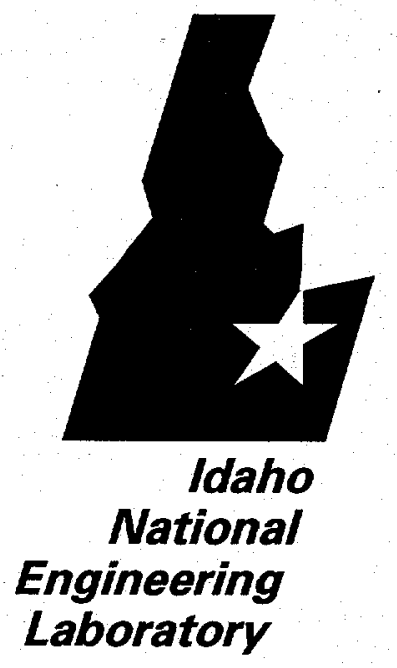

INEL-95/0234

RECEIVED

October 1995

NOV 211995

USTI

Practical Guidance for Statistical Analysis of Operational Event Data

Corwin L. Atwood

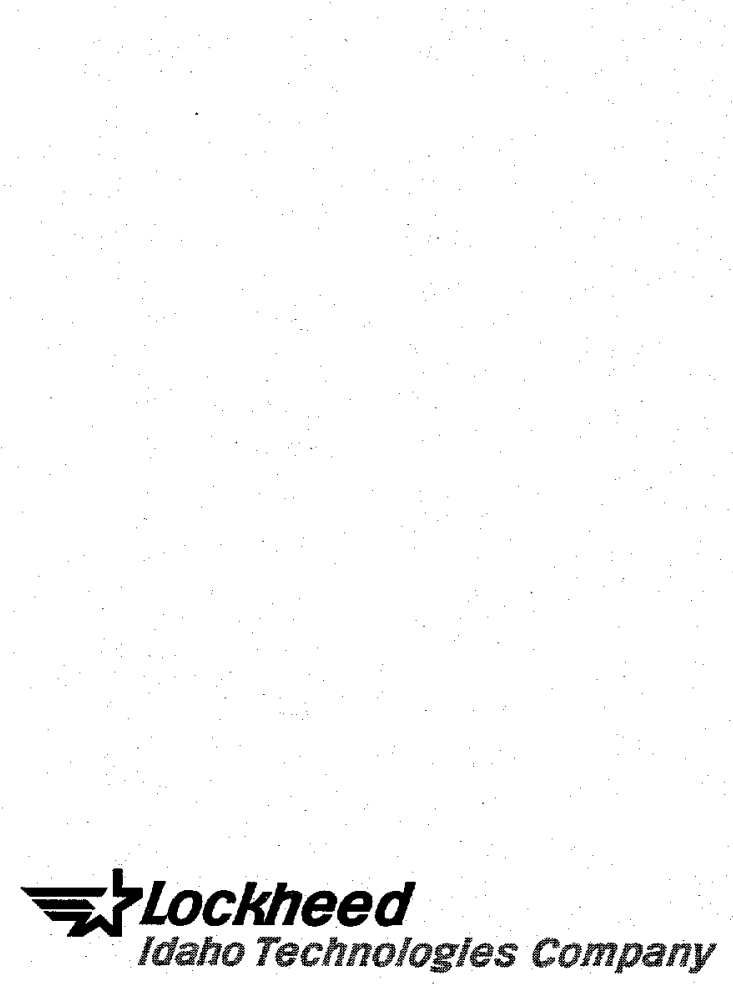




\section{NOTICE}

This report was prepared as an account of work sponsored by an agency of the United States Government. Neither the United Sates Government nor any agency thereof, nor any of their employees, makes any warranty, expressed or implied, or assumes any legal liability or responsibility for any third party's use, or the results of such use, of any information, apparatus, product or process disclosed in this report, or represents that its use by such third party would not infringe privately owned rights. 
This report was prepared as an account of work sponsored by an agency of the United States Government. Neither the United States Government nor any agency thereof, nor any of their employees, makes any warranty, express or implied, or assumes any legal liability or responsibility for the accuracy, completeness, or usefulness of any information, apparatus, product, or process disclosed, or represents that its use would not infringe privately owned rights. Reference herein to any specific commercial product, process, or service by trade name, trademark, manufacturer, or otherwise does not necessarily constitute or imply its endorsement, recommendation or favoring by the United States Government or any agency thereof. The views and opinions of authors expressed herein do not necessarily state or reflect those of the United States Government or any agency thereol.

\title{
Practical Guidance for Statistical Analysis of Operational Event Data
}

\author{
Corwin L. Atwood
}

Published October 1995

\section{Idaho National Engineering Laboratory Applied Engineering Development Laboratory Lockheed Martin Idaho Technologies Idaho Falls, Idaho $\mathbf{8 3 4 1 5}$}

\author{
Prepared for the \\ U.S. Nuclear Regulatory Commission \\ Office for Analysis and Evaluation of Operational Data \\ Reliability and Risk Analysis Branch \\ Under DOE Idaho Operations Office \\ Contract DE-AC07-94ID 13223
}




\section{DISCLAIMER}

Portions of this document may be illegible in electronic image products. Images are produced from the best available original document. 


\begin{abstract}
This report presents ways to avoid mistakes that are sometimes made in analysis of operational event data. It then gives guidance on what to do when a model is rejected, a list of standard types of models to consider, and principles for choosing one model over another. For estimating reliability, it gives advice on which failure modes to model, and moment formulas for combinations of failure modes. The issues are illustrated with many examples and case studies.
\end{abstract}




\section{SUMMARY}

This report gives practical guidance for analyzing operational event data. The report begins by presenting mistakes that are sometimes made in analysis of operational event data, and gives ways to avoid them. It reminds the analyst to normalize count data correctly, both by using the correct denominator for a ratio and by asking the right questions, questions that are directly related to the process that produced the data. To help account for random variation in the data, it recommends displaying error bars in the presentations. It explains how to account for the performance of many simultaneous tests. It explains Simpson's paradox, and the dangers of looking at the explanatory variables one at a time.

The report then gives guidance for what to do when a relatively simple model has been rejected: identify the features of the data that caused the model to be rejected, talk with subject-matter experts and formulate hypotheses, and try to test these hypotheses and decide on a better model.

As an aid to building a better model, the report lists standard types of models that the statistical analyst should be familiar with, including homogeneous populations with outliers, empirical Bayes models, linear models, and loglinear and logit models. The report suggests principles for choosing one model over another: understanding of the true natural process, parsimony, economy of display, and goodness of fit.

Finally, in the context of reliability estimation, the report gives guidance on which failure modes to model: model only what is necessary, and avoid modeling what cannot be estimated well. Split the data into groups only if the different groups correspond to different parameter values. The report shows how to use a constrained noninformative prior to minimize the worst effects of dividing the data too finely, if the customer requires data to be split anyway. When combining failure modes, such as when computing unreliability, use Monte Carlo simulation from the posterior distributions, or algebraic formulas for the moments. SAS implementations of these formulas are given in an appendix.

The issues are illustrated by many case studies and examples. The report concludes with a short review. 


\section{FOREWORD}

The Office for Analysis and Evaluation of Operational Data (AEOD) of the U.S. Nuclear Regulatory Commission (NRC) is continually reviewing data from U.S. commercial nuclear power plants. Statistical analysis forms an important part of this work. Accordingly, the Reliability and Risk Analysis Branch of AEOD has asked the Idaho National Engineering Laboratory (INEL) to write a series of reports presenting the appropriate statistical tools for the kinds of data most commonly encountered. These reports are being written in parallel with a series of studies on the performance of safety systems in nuclear power plants, and they reflect the influence of those studies.

The reports are expected to provide tools and guidance to analysts of NRC data, although the statistical methods can, by their nature, be applied to data from many other fields. The reports are intended to quickly help a new user. The report on collecting operational data should be understandable by anyone with a technical background, although the examples are slanted towards an engineer with nuclear experience. The reports on statistical methods should be readable and immediately usable by a person with training in statistics but with no experience analyzing such data. Some of the reports, of necessity, are more advanced than others; for example, the report on loglinear modeling will be easier to understand if the reader has first assimilated the reports on binomial data, Poisson data, and linear models. Nevertheless, the reports are all intended to be introductory to the extent possible, suitable as brief self-study texts to help readers move quickly to the tasks of data collection and analysis. In addition, the reports should be usable as texts or references in short courses for persons with less training.

The first reports written or planned in this series are

Collecting Operational Event Data for Statistical Analysis, September 1994, EGG-RAAM-10086, by Corwin L. Atwood

Hits per Trial: Basic Analysis of Binomial Data, September 1994, EGGRAAM-11041, by Corwin L. Atwood

Events in Time: Basic Analysis of Poisson Data, September 1994, EGGRAAM-11088, by M. E. Engelhardt

Modeling Patterns in Continuous Data Using Linear and Related Models DRAFT, 1995, INEL-95/0120, by M. E. Engelhardt

Modeling Patterns in Count Data Using Loglinear and Related Models, to appear 1995, INEL-95/0121, by Corwin L. Atwood 
Statistical Analysis of Random Duration Times DRAFT, 1995, INEL95/0206, by M. E. Engelhardt

Practical Guidance for Statistical Analysis of Operational Event Data, October 1995, INEL-95/0234, by Corwin L. Atwood 


\section{ACKNOWLEDGMENTS}

Mark DeHaan, Max Engelhardt, Cindy Gentillon, and Harry Martz reviewed a draft of this report, and each made suggestions that significantly improved both the content and the presentation. 


\section{CONTENTS}

ABSTRACT $\ldots \ldots \ldots \ldots \ldots \ldots \ldots \ldots \ldots \ldots \ldots \ldots \ldots \ldots \ldots \ldots \ldots \ldots \ldots$

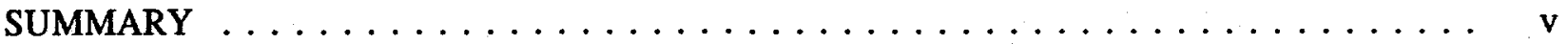

FOREWORD $\ldots \ldots \ldots \ldots \ldots \ldots \ldots \ldots \ldots \ldots \ldots \ldots \ldots \ldots \ldots \ldots$ vii

ACKNOWLEDGMENTS $\ldots \ldots \ldots \ldots \ldots \ldots \ldots \ldots \ldots \ldots \ldots \ldots \ldots \ldots$

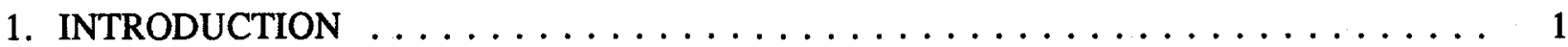

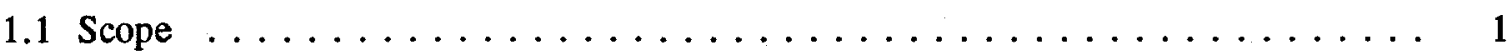

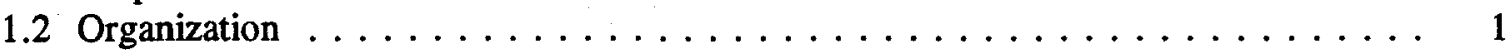

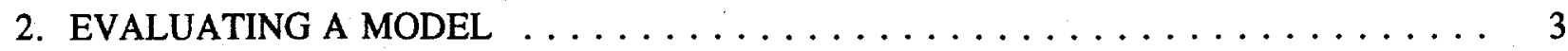

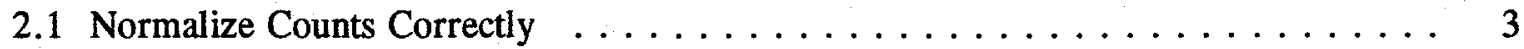

2.1.1 Using the Right Denominator $\ldots \ldots \ldots \ldots \ldots \ldots \ldots$

2.1.2 Asking the Right Question .................. 6

2.2 Consider Uncertainties When Making Inferences $\ldots \ldots \ldots \ldots \ldots \ldots$

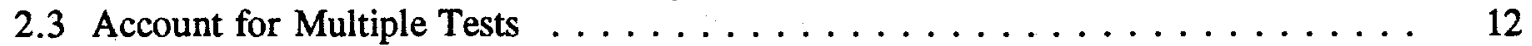

2.3 .1 Basic Facts . . . . . . . . . . . . . . . . . 12

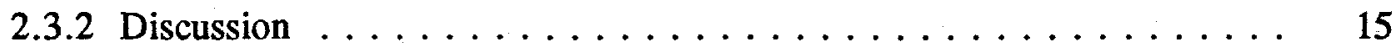

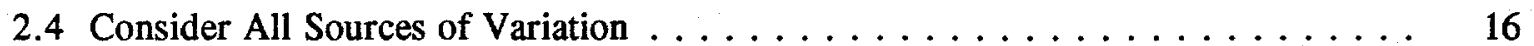

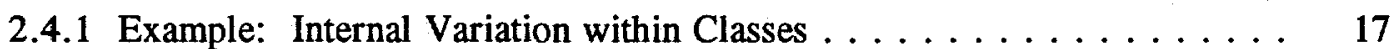

2.4.2 Example: Ignoring an Explanatory Variable . . . . . . . . . . . 17

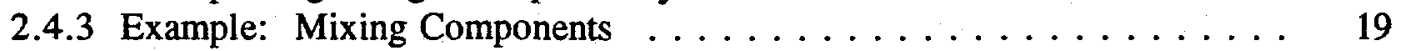

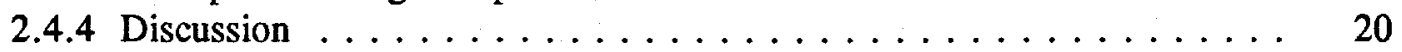

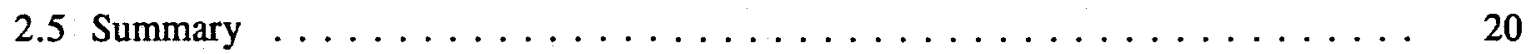

3. WHAT TO DO WHEN A MODEL IS REJECTED $\ldots \ldots \ldots \ldots \ldots \ldots \ldots \ldots$

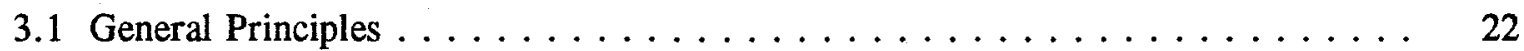

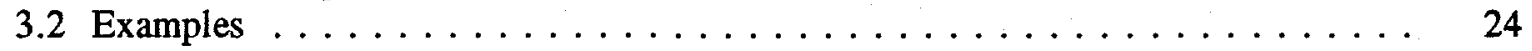

3.2.1 Air Conditioners That Appeared to Improve with Time . . . . . . . . . 24

3.2.2 Between-Plant Variation of Diesel Generator Failure Rates . . . . . . . 25

3.2.3 Between-Plant Variation for HPCI Failures to Start . . . . . . . . . . 25

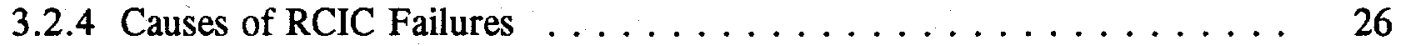

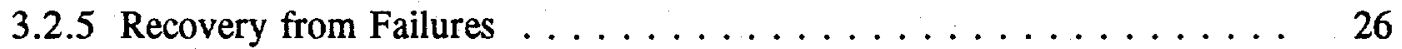

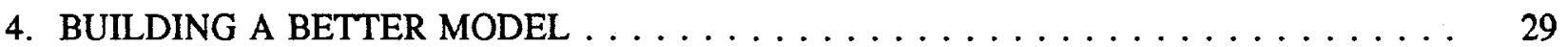

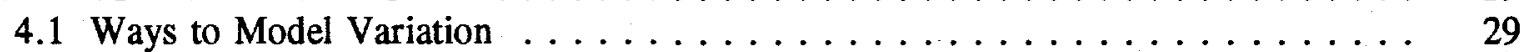

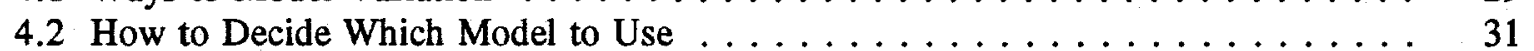

4.2.1 Understanding $\ldots \ldots \ldots \ldots \ldots \ldots \ldots \ldots \ldots \ldots \ldots \ldots$

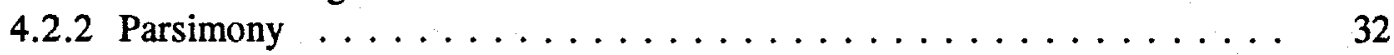

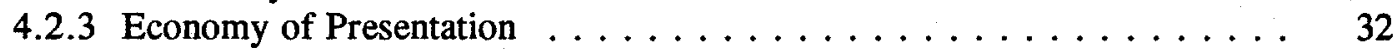

4.2 .4 Goodness of Fit $\ldots \ldots \ldots \ldots \ldots \ldots \ldots \ldots \ldots \ldots \ldots$

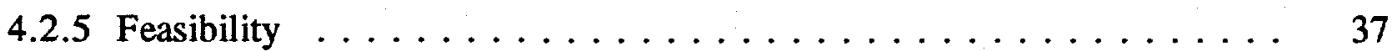

5. CHOOSING AND COMBINING FAILURE MODES $\ldots \ldots \ldots \ldots \ldots \ldots$

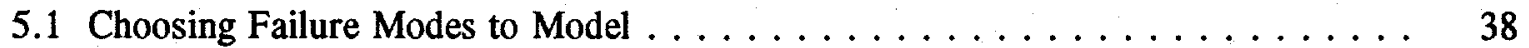

5.1.1 Subdivide Failure Modes Only as Necessary . . . . . . . . . . 38 


\subsubsection{Try to Avoid Estimating Failure Probabilities when No Failures}

Occurred ........................ 40

5.1.3 Do Not Estimate Quantities for Which No Data Exist . . . . . . . 40

5.1.4 Split Data into Subsets Only as Necessary . . . . . . . . . . . . . 41

5.1 .5 Case Study: HPCS System . . . . . . . . . . . . . . . 43

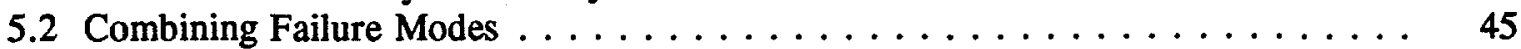

5.2.1 Formulas Using Moments, for Probabilities of Failures on Demand . . . 45

5.2.2 Formulas Using Moments, for Rates of Events in Time . . . . . . . . 47

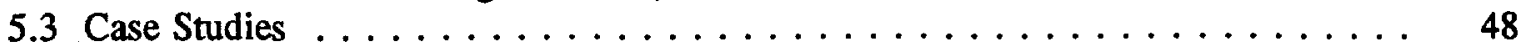

5.3.1 The AFW System . . . . . . . . . . . . . . . . . 48

5.3.2 Use of Constrained Noninformative Prior When Data Are Split into

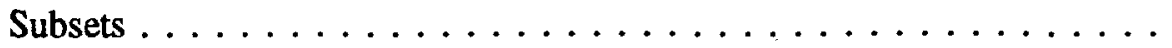

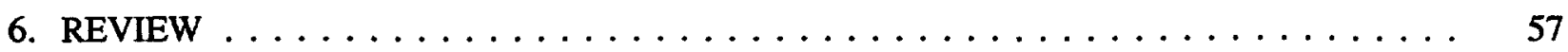

6.1 Data Analysis for Evaluating an Explicit or Implicit Model . . . . . . . . 57

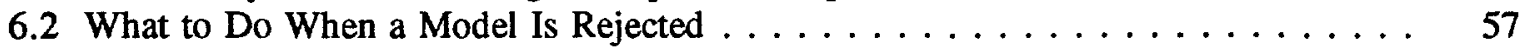

6.3 Building a Better Model . . . . . . . . . . . . . . . . . 57

6.4 Choosing and Combining Failure Modes $\ldots \ldots \ldots \ldots \ldots \ldots \ldots$

7. REFERENCES $\ldots \ldots \ldots \ldots \ldots \ldots \ldots \ldots \ldots \ldots \ldots \ldots \ldots \ldots$

APPENDIX A-SAS PROGRAMS $\ldots \ldots \ldots \ldots \ldots \ldots \ldots \ldots \ldots \ldots \ldots \ldots$

APPENDIX B-LIKELIHOOD FORMULAS $\ldots \ldots \ldots \ldots \ldots \ldots \ldots \ldots$

\section{FIGURES}

1. Number of events caused by aging versus years of operation. Failure to normalize leads to incorrect interpretation of this graph. . . . . . . . . . . . . . . .

2. Rate of events caused by aging, defined as number of such events in year $i$ divided by number of plants contributing to year $i$.

3. Normalized failure frequency for valve operators as a function of age at failure. The stated normalizer is incorrect.

4. Fraction of RCIC component failures that caused lost or degraded system function. This hides whether the change is in the numerator, denominator, or both.

5. Graphical display of Table 1 , two hypothetical data sets that could produce Figure $4 . \ldots \ldots$

6. Types of RCIC failures, by plant age group. This does not show which failure types behave differently in the two age groups.

7. HPCI inoperabilities per plant operational year, with $90 \%$ confidence intervals. The error bars help a reader draw valid inferences. . . . . . . . . . . . . . . .

8. Venn diagram with example events. Shaded region is union.

9. Observed mortality versus prenatal care and clinic. Dot size suggests amount of data. Dashed line results from (mistakenly) pooling clinics. . . . . . . . . . . . . . .

10. Combined failure frequency for seven components. Different components may dominate different portions of the graph. . . . . . . . . . . . . . . . . .

11. Probabilities of various failure modes, for unplanned actuation (A) and surveillance test (T) data sets.

12. Failure rates for pressure switches-Bayes means and $90 \%$ intervals. . . . . . . . . . 33

13. Hypothetical failure rates-Bayes means and $90 \%$ intervals. . . . . . . . . . . 34 
14. Fault tree for failure to operate of single AFW train, excluding common-cause failures.

15. Unreliability of a typical AFW system.

16. Posterior means and $90 \%$ intervals for unreliability, using the Jeffreys noninformative prior and the constrained noninformative priors.

\section{TABLES}

1. Two hypothetical data sets that could produce Figure $4 . \ldots \ldots \ldots \ldots \ldots \ldots$

2. Types of RCIC failures, by plant age group.

3. Number of RCIC failures divided by plant operating years, by failure cause and plant age

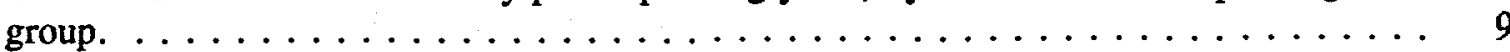

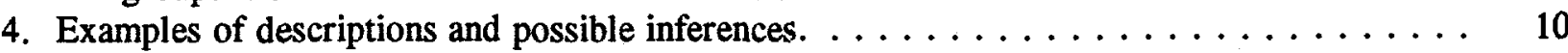

5. Prenatal care versus infant mortality. . . . . . . . . . . . . . . . . . . 19

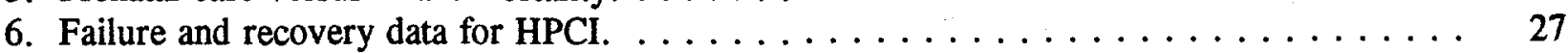

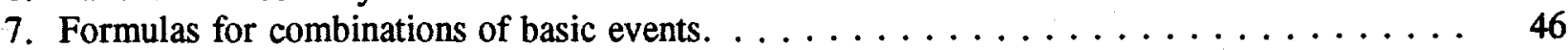

8. SAS macro for train unreliability, ignoring common-cause failures. . . . . . . . . 50

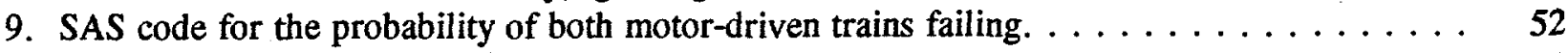

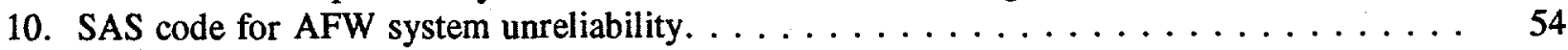




\section{Practical Guidance for Statistical Analysis of Operational Event Data}

\section{INTRODUCTION}

\subsection{Scope}

This report discusses some of the practical aspects of the statistical analysis of operational event data. Event data consist of information describing observed occurrences of events of interest; although portions of this report are applicable to any data, all the examples presented involve events, typically failures. Operational data are data that were collected from a process whose primary purpose was not merely the generation of the data. An important example is data collected during operation of a power plant. Because the data do not come from a neatly balanced and well-designed experiment, difficulties of interpretation can arise.

The report is part of a series, as described in the foreword. Most of the other reports in the series present statistical techniques for analyzing specific kinds of data. This report has a different emphasis, a concern with the overall approach to an analysis and with avoidance of conceptual errors. To best appreciate the points made, a reader should have some familiarity with statistical analysis of event data. Where specific technical knowledge is required, this report gives references.

The issues are illustrated by many short examples, often from work at the INEL but also from other studies, published and unpublished. Some of these examples illustrate mistakes. The sources of such examples are not given, because the purpose is not to pillory other work but to learn from those mistakes. Nevertheless, the examples actually appeared in print, either in draft or final reports; they are not made up unless that fact is stated. In addition to object lessons, this report attempts to give guiding principles where appropriate. It suggests some commonly used models, and gives some principles for choosing among possible models. Because many of the envisioned applications involve reliability and probabilistic risk assessment, modeling of failure modes is discussed specifically as a separate topic.

\subsection{Organization}

Data analysis usually begins with some extremely simple model, typically the model that all the observations are independent and have the same simple distribution. This model may not be explicitly stated, but it is usually in the back of the analyst's mind. The analyst evaluates the model, through graphical techniques, descriptive tables, and formal statistical tests of hypotheses. If the model is extremely simple, these techniques may involve nothing more than descriptions of the data. For example, 
plotting data to look for a trend can be regarded as informally testing the model of no trend, and it is regarded in this way for the purpose of organizing this report. This evaluation or testing activity may lead to rejection of the model, and the tentative formulation of a different model. Then the process begins anew with critiques of this model. Ultimately, the model-building process should terminate. Then the analyst uses the selected model to calculate quantities of interest, such as a failure probability or a reliability, with appropriate uncertainty intervals.

The organization of this report follows the above process. Section 2 presents mistakes that can be made when using graphical plots or statistical tests to evaluate a model. These mistakes are ordered from the simple to the subtle. Section 3 discusses what to do when a model is rejected. This leads into Section 4, containing a treatment of possible models in the analyst's toolbox, and principles for choosing among models. Section 5 considers estimation of reliability, based on combining failure modes. The main points of the report are reviewed in Section 6. Section 7 contains references. Two short appendices are provided, one with some computer macros and one with some formulas useful in calculation of likelihoods. 


\section{EVALUATING A MODEL}

This section considers mistakes that can be made in examining data or a model of the datageneration process. The mistakes are ordered from the most blatant to the most subtle. Two other discussions of this type are Agresti (1983) and Long (1988).

\subsection{Normalize Counts Correctly}

Counts alone often are not very informative. Instead, they must be normalized, divided by some number to yield a fraction that is meaningful. For example, when considering some kind of failure, the probability of failure is estimated by the observed number of failures divided by the number of demands. Similarly, the rate of failures per plant-year is estimated by the observed number of failures divided by the number of plant-years. Other fractions can also be calculated, but fractions that represent probabilities or rates are typically the easiest to interpret, because the probability or rate is directly related to the underlying process that produced the data.

Meaningful normalization involves two steps, therefore:

- Deciding on the real quantity of interest-asking the right question

- Using the appropriate denominator to estimate the desired quantity

When a misleading or incorrect analysis is performed, it may be hard to say whether the analyst asked the wrong question or answered the right question badly. Nevertheless, the examples below are grouped according to the two steps. The second step is easier, so it is considered first.

\subsubsection{Using the Right Denominator}

When the quantity of interest is the probability of failure on demand, the denominator should be the number of trials. That is, the numerator is a count of failures, and the denominator should be the number of occasions when such failures could occur. For a rate of events in time, the denominator should be the total time when the events in the numerator could possibly occur. The mistake considered in this section is use of the wrong denominator. For example, a system that is powered by a steam turbine, such as the high-pressure coolant injection (HPCI) system in a boiling water reactor, can be demanded only when steam is being generated. It would be a mistake to estimate the rate of HPCI demands as (number of demands in a year)/(number of hours in a year). The denominator should instead be (number of hours in a year when steam was generated). 
A special case arises when an investigator compares counts without normalizing them at all. This is equivalent to setting the denominator equal to 1 , which may be an incorrect normalizer. For example, more people of age 50 die each year than do people of age 100 . Does that mean that a 50 -year old is at greater risk than a 100-year old? No, only that the population has more 50-year olds. If the quantity of interest is the probability of death for a person of a certain age, the observed probabilities should be compared:

(number of deaths of people of age $i$ ) / (number of people of age $i$ in the population) .

This fraction estimates the probability that someone of age $i$ will die during the year, and can be used to compare the relative risk for people of different ages.

Figure 1 shows a similar example. Based on (apparently) all the nuclear power reactors in one country, the figure shows the number of events caused by aging (such as wear or corrosion) grouped by age of the plant. The draft report from which the plot was taken comments, "It can be seen that more events are experienced in less years of operation and the frequency of events decreases as the number of years of operation increases. In this regard, it is considered that the so-called early failures tend to occur due to a combination of defects in design, fabrication, installation and maintenance, operating conditions and operating environments."

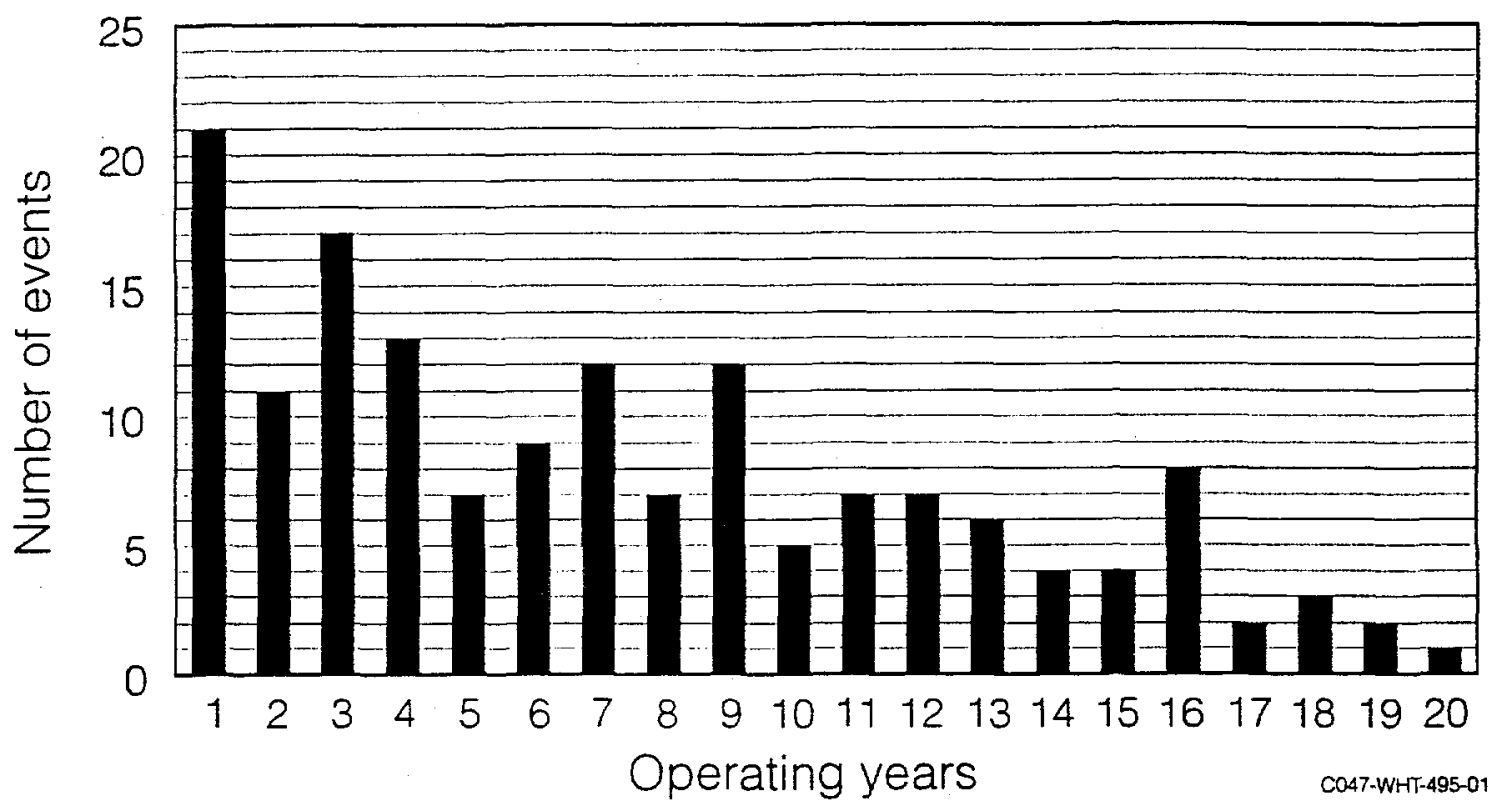

Figure 1. Number of events caused by aging versus years of operation. Failure to normalize leads to incorrect interpretation of this graph. 
The graph is misleading, and it led the writers to a mistaken conclusion. It is true that fewer events were reported for plants in their twentieth year than for plants in their first year. However, every plant has gone through its first year, and few have gone through the twentieth year. The graph should have plotted not the numbers but the observed rates, defined as

(number of events in year $i$ ) / (number of plants contributing data from year $i$ ) .

When this was pointed out to the authors of the draft report, they immediately added a line to the plot showing the estimated rate for plants of each age. Figure 2 shows this line as a separate graph, although the authors of the report simply added it on top of the bars in Figure 1.

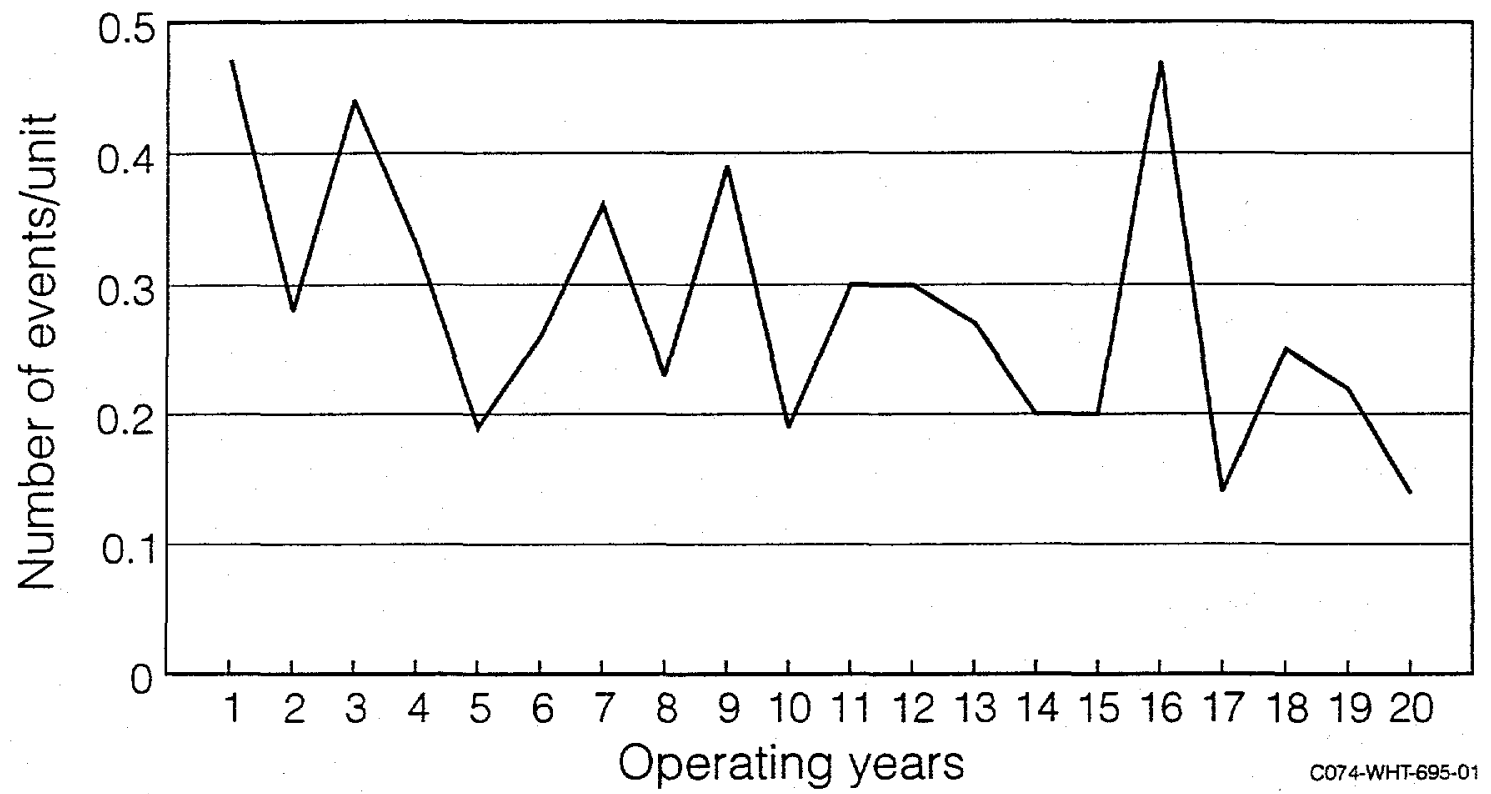

Figure 2. Rate of events caused by aging, defined as number of such events in year $i$ divided by number of plants contributing to year $i$.

Figure 3, from a different report, explicitly uses a normalizer, but it is the wrong one. The figure claims to show the rate of failures of valve operators, as a function of age at failure. The accompanying text says it plots "the number of valve operator failures per unit-year as a function of component age, normalized for 26 plants." This division of all the counts by 26 simply rescales the vertical axis. The quantity that should be studied is the rate of failures per valve-operator-year. Because the number of valve operators of each age varies, for age $i$ the denominator should be the number of valve operators of age $i$ for which failures would be reported. However, the text and the axis label state that the denominator is the number of unit-years. This is a mistake. 


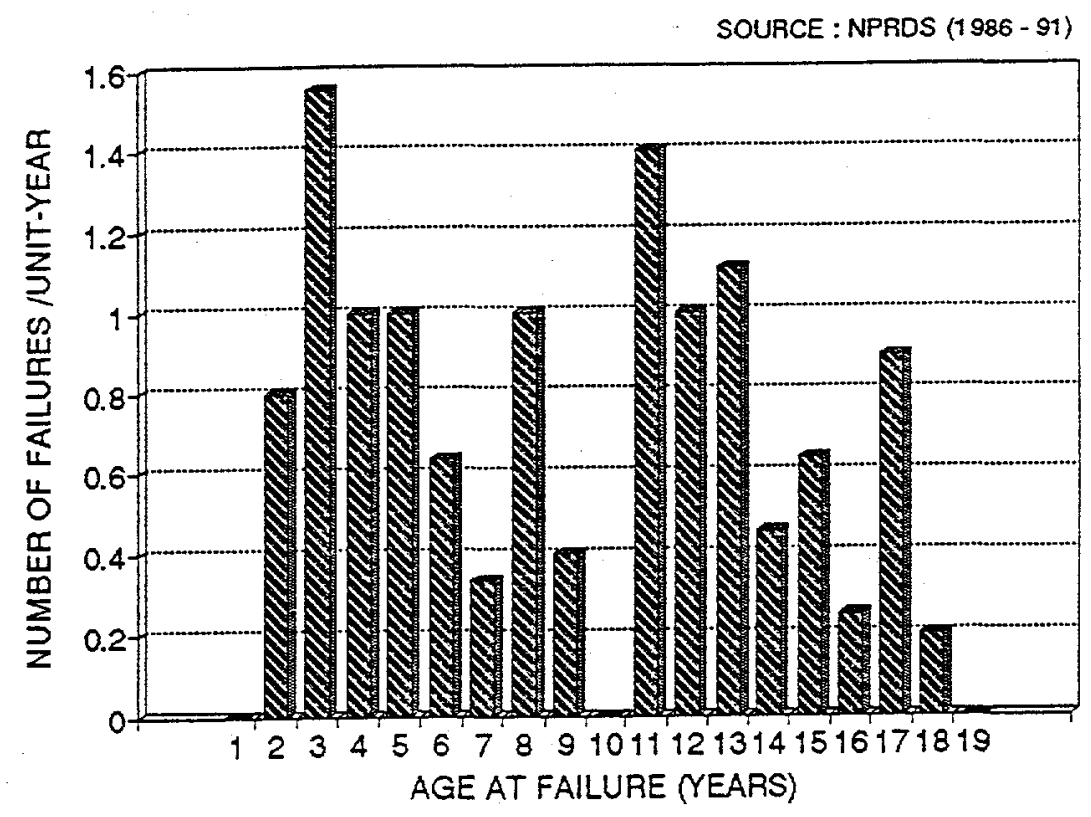

Figure 3. Normalized failure frequency for valve operators as a function of age at failure. The stated normalizer is incorrect.

\subsubsection{Asking the Right Question}

The most meaningful quantities are those that are directly related to the process that generated the data, such as event rates and failure probabilities. When questions are asked about such quantities, the answers are easy to interpret, and yield insight into the process. Questions about other quantities may result in answers that are hard to interpret or downright misleading.

To illustrate this, consider Figure 4, taken from the same report that contains Figure 3. It shows the fraction of reactor core isolation cooling ( $\mathrm{RCIC}$ ) component failures that seriously affected system operation. The accompanying text states, "About the same number of plants reported component failures between January 1986 and December 1991, so it is clear that the effects of the component failures become more and more serious as the components age." Let us ignore the implicit assumption that the components in later years are older than the components in earlier years; let us also ignore the question of whether the observed trend is a result of chance alone or is a reflection of the underlying process; instead, let us simply ask what is driving the trend. Are the serious failures per component-year increasing, or are the nonserious failures per component-year decreasing, or are both changing but at different rates? The graph gives no information to answer this question.

For example, the two sets of made-up data in Table 1 and Figure 5 show failure counts per time period. They tell very different stories about the serious failures but they both give the fractions in Figure 4. In the first case, serious failures are becoming more frequent and nonserious failures less 


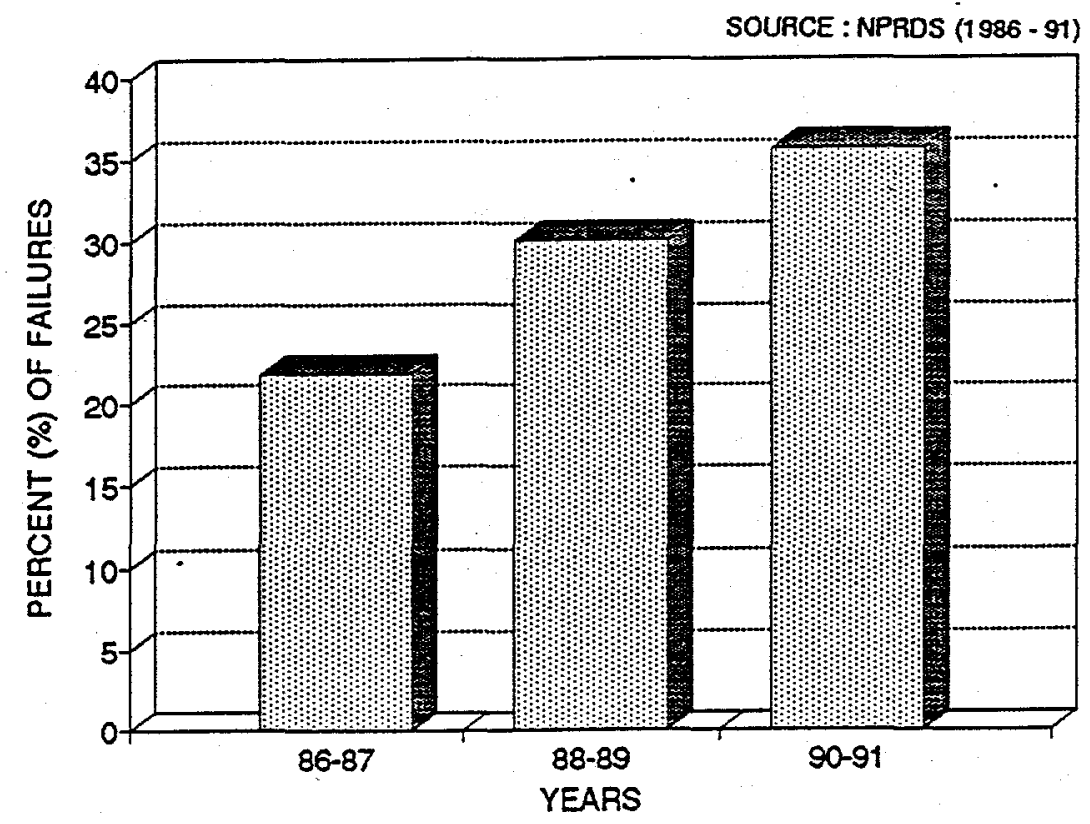

Figure 4. Fraction of RCIC component failures that caused lost or degraded system function. This hides whether the change is in the numerator, denominator, or both.

Table 1. Two hypothetical data sets that could produce Figure 4.

\begin{tabular}{lllllllll}
\hline & \multicolumn{3}{c}{ First Data Set } & & \multicolumn{3}{c}{ Second Data Set } \\
\cline { 2 - 7 } & $\underline{86-87}$ & $\frac{88-89}{30}$ & $\frac{90-91}{36}$ & & $\frac{86-87}{30}$ & $\frac{88-89}{30}$ & $\frac{90-91}{30}$ \\
Number of serious failures & 78 & 70 & 64 & & 106 & 70 & 53 \\
Number of nonserious failures & $22 \%$ & $30 \%$ & $36 \%$ & & $22 \%$ & $30 \%$ & $36 \%$ \\
Fraction of serious failures & & & & & & & \\
\hline
\end{tabular}

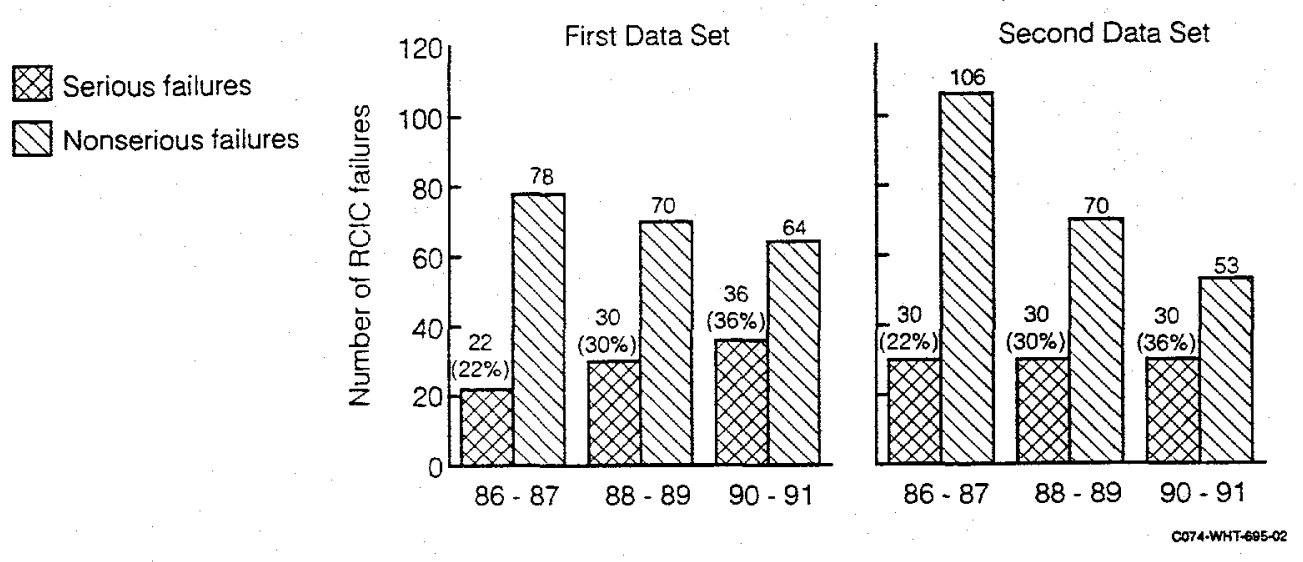

Figure 5. Graphical display of Table 1, two hypothetical data sets that could produce Figure 4. 
frequent. In the second case the serious failures are occurring at a constant rate and, for some reason, fewer nonserious failures are being reported as time goes on.

The report was asking the wrong question. It is true that the observed fraction of serious failures was increasing, but so what? The rate of serious failures and the rate of nonserious failures would have been much more meaningful quantities to estimate. They are related directly to the process that produces the failure reports. Moreover, one could calculate the fraction of failures that are serious from the rates of the two kinds of failures.

Such an analysis was performed in an unpublished INEL draft study of the RCIC system. (This study was unrelated to the RCIC study from which Figures 3 and 4 were taken.) The causes of failures were tallied separately for older and newer boiling water reactors (BWRs). This tally is shown in Table 2. A pair of pie charts of the percentages (Figure 6) made it appear that the primary difference was in the fraction of personnel errors.

Table 2. Types of RCIC failures, by plant age group.

\begin{tabular}{lccc} 
& Equipment & Personnel & Procedure \\
\cline { 2 - 3 } Older BWRs & $39(83 \%)$ & $7(15 \%)$ & $1(2 \%)$ \\
Newer BWRs & $20(71 \%)$ & & $7(25 \%)$
\end{tabular}

Older BWRs
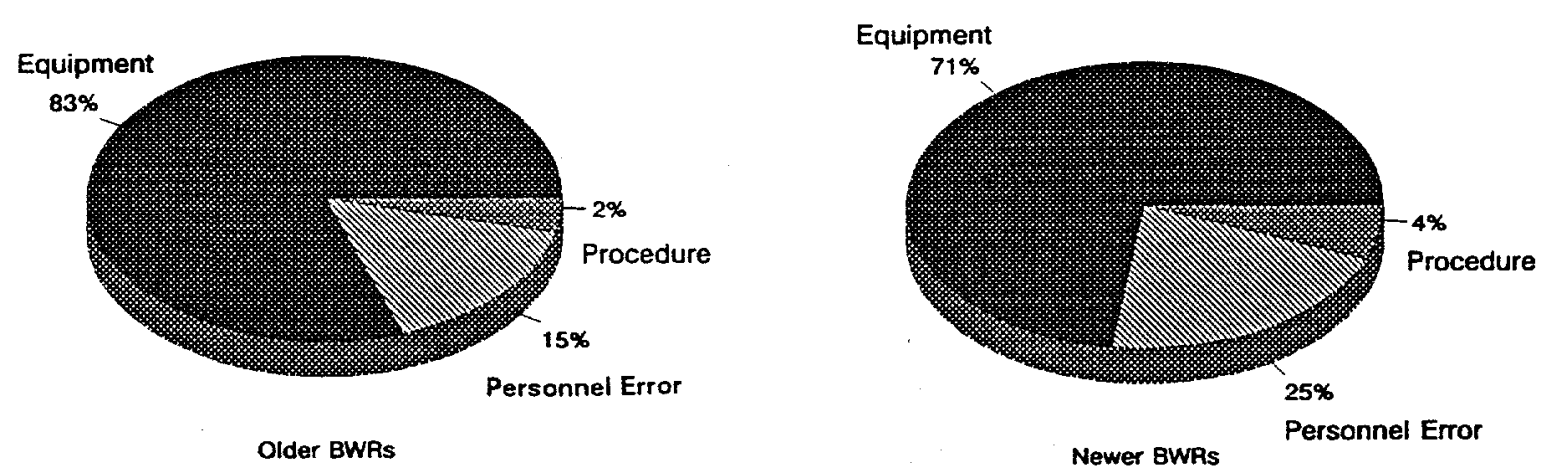

Figure 6. Types of RCIC failures, by plant age group. This does not show which failure types behave differently in the two age groups.

The authors of the study investigated the data to better understand the differences seen. They normalized the events by operating year, where the number of operating years for a BWR is the total time, in years, when the reactor was at power. This version of the data is shown in Table 3. The operating years for a group of plants is the sum of the operating years for the plants in the group. This 
led to estimated failure rates per operating year. For failures from personnel errors, the rates were 0.214 failures/yr for the older BWRs and 0.207 failures/yr for the newer BWRs, virtually the same. The failure rates from equipment were 0.636 failures/yr for the older BWRs and 0.315 failures/yr for the newer BWRs. These differ by a factor of two. $90 \%$ confidence intervals for the two rates do not overlap, and when a statistical test was performed (see Engelhardt 1994), the difference was statistically significant with $p$-value $=0.0093$. (This $p$-value cannot be taken at face value, as discussed below in Section 2.3, but for the moment we accept it.)

Table 3. Number of RCIC failures divided by plant operating years, by failure cause and plant age group.

\begin{tabular}{llll} 
& Equipment & Personnel & Procedure \\
\cline { 2 - 3 } Older BWRs & $39 / 61.36$ & $7 / 61.36$ & $1 / 61.36$ \\
Newer BWRs & $20 / 63.42$ & & $7 / 63.42$
\end{tabular}

Therefore, the two classes of reactors do indeed behave differently, but the difference does not involve personnel errors. Instead, equipment failures are about twice as frequent in the older plants as in the newer plants. The investigation then focused on possible reasons for this difference.

The authors' initial desire to summarize the data in a small number of pie charts led them to calculate row percentages, obscuring the real difference between the data sets. If Table 2 had shown column percentages instead of row percentages, the difference would have been more evident from the start: $39 / 59=66 \%$ of the equipment failures occurred in older BWRs, compared to $50 \%$ of each other failure type in older BWRs. But this visible difference would have been a result of luck, not method; the different percentages would have reflected different failure rates because the plant operating years are almost the same in the two rows. If the operating years for older and newer BWRs had differed substantially, column percentages would produce a misleading result. The problem is in computing percentages of failure counts, however it is done, instead of computing rates of failures per time unit.

This discussion can be summarized as follows. The fundamental question is not "Do the two groups of reactors have the same fractions of failures for each category?” Discovering that they have different fractions does not reveal whether one group of reactors is low for one failure category or high for a different failure category. A much more informative question is "Do the two groups of reactors have the same failure rate for each category?" This question is directly related to the processes that produce the failures, and focuses on identifying any problem areas. 
As a side issue, we point out that Table 2 , when analyzed as a $2 \times 3$ contingency table, shows no significant dependence between plant age and failure type. The Pearson $\chi^{2}$ statistic equals 1.395 , near the median of the $\chi^{2}$ distribution with 2 degrees of freedom. Why does the test based on counts alone (Table 2) find no difference between the plant type and failure type, although the test based on rates (Table 3) reaches different conclusions for the three kinds of failure type? The tests are testing different hypotheses. The test using Table 3 tests whether the two classes of plants have equal failure rates, for each type of failure, by comparing the failure counts of each type (such as the equipment failure counts) to nonrandom times. The contingency table test, on the other hand, asks if the two classes of plants have the same fractions of failure of each type. It performs the test by comparing the number of failures of each type, such as equipment failure counts, to the random counts for the other failure types. The comparison to nonrandom quantities seems to give a firmer basis for a conclusion than the comparison to random quantities.

\subsection{Consider Uncertainties When Making Inferences}

It is perfectly valid to tabulate or plot numbers from the data, to show what happened. However, an analyst then typically wants to draw inferences, to say something about the process that generated the data. The distinction is illustrated in Table 4.

Table 4. Examples of descriptions and possible inferences.

\section{Description}

A component failed twice in twenty demands.

The number of failures per component were 0.015 , $0.012,0.013,0.009$, and 0.010 in five successive years.

Diesel generators had 0.092 failures per demand in BWRs and 0.087 failures per demand in PWRs.

\section{Possible Inference}

The probability of failure is $2 / 20=0.10$.

There is a downward trend over time.

Diesel generators fail less often in PWRs than in BWRs.

The descriptions in Table 4 state what happened, in the past tense. The inferences, on the other hand, make statements about the underlying process, and the verbs are in the present tense because they describe properties of nature. In the language of statistics, the descriptions are statements about samples, and the inferences are statements about populations.

The data do not immediately reveal how much of what happened was chance and how much was an underlying pattern. To make an inference based on the data, the analyst must consider the random nature of the data, and quantify the uncertainty in the inference. For example, Figure 2 does not show 
the uncertainty in the estimated rates. However, the authors of the report do not attempt to draw inferences from the line.

The report containing Figures 3 and 4 makes the error of not considering the uncertainties when making inferences. The figures do not show uncertainty intervals, and the report text treats the high and low values seen as a result of the underlying process, not as possibly a result of chance. Regarding Figure 3, the report text worries about the causes of the peaks and why the failure frequency decreases after the peaks. Before addressing such concerns, the report should have established that the peaks are really present in the underlying process and are not merely random bounces in the data.

To show the uncertainties, bar charts should not be used. The solid bars in Figures 1, 3, and 4 get in the way of uncertainty intervals. Instead, use dots to show the data values, and draw an error bar around each dot, showing the relevant uncertainty for inferences. Figure 7, adapted from Grant et al. (1995), is such a graph. For each calendar year, the reported number of HPCI inoperabilities was divided by the number of plant operational years (the time when such events could occur) to produce the observed rate. Each observed rate was plotted, and a $90 \%$ confidence interval for the underlying true rate was also shown. The confidence intervals were based on the assumption that the counts followed a Poisson distribution, as described, for example, by Engelhardt (1994).

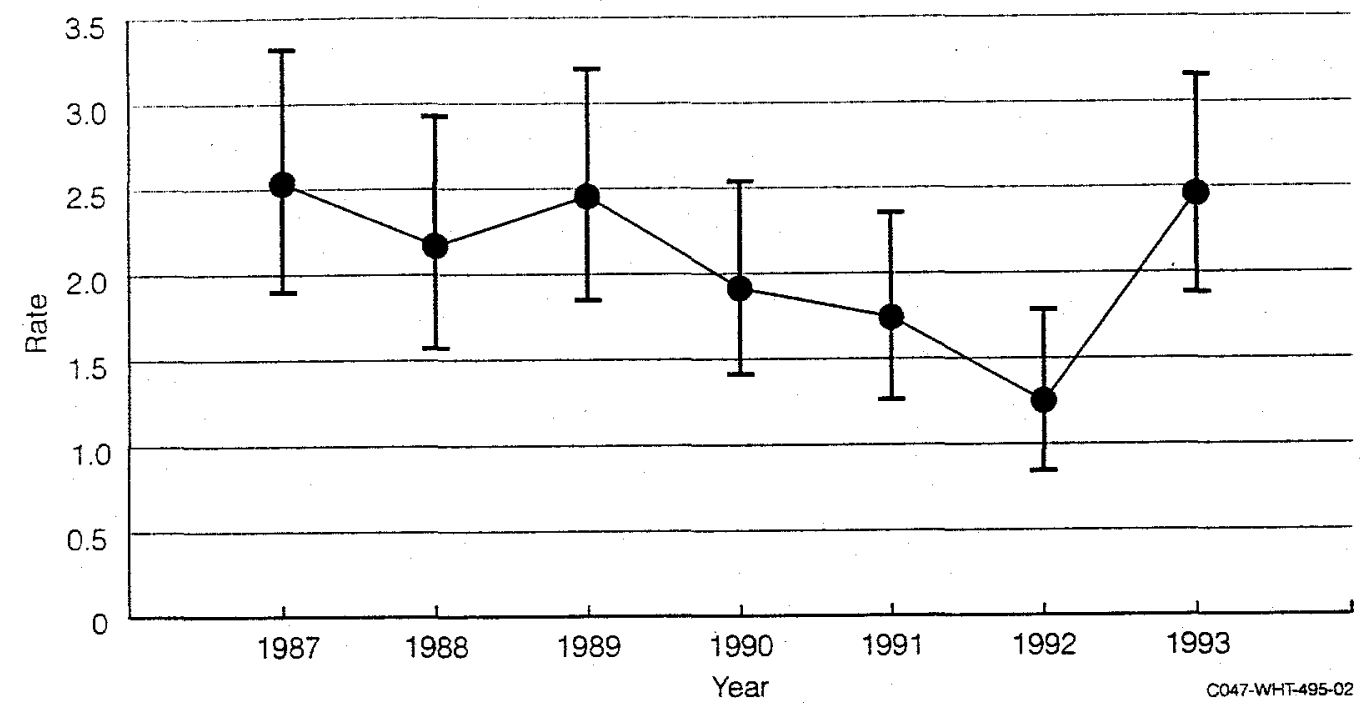

Figure 7. HPCI inoperabilities per plant operational year, with $90 \%$ confidence intervals. The error bars help a reader draw valid inferences.

Because the error bars largely overlap, it is evident that some or all of the variability in the line may be attributed to chance. Before the 1993 data had been collected, the plot without error bars would have appeared to be trending downward. Adding the error bars to the plot would have shown that the 
apparent trend may be the result of chance alone. The jump in 1993 would be much less surprising to someone who had seen the error bars for the earlier years.

\subsection{Account for Multiple Tests}

\subsubsection{Basic Facts}

As mentioned in connection with Table 3, the test based on (number of equipment failures)/(plant operating years) rejects the hypothesis of equal rates for older and newer BWRs. The $p$-value is 0.0093 . Three such hypotheses could be tested, corresponding to failures caused by equipment, failures caused by personnel, and failures caused by procedural errors. The only reason for focusing on equipment failures was that they showed the greatest difference in Table 3. The analysis should account for this data-driven choice of the hypothesis to test.

A postulated hypothesis, $H_{0}$, may be rejected, not because it is false, but simply because of the randomness of the data. To reject $H_{0}$ at the 5\% significance level is to say, "If $H_{0}$ is true, the chance that the data would be this extreme is only 5\%." Even if $H_{0}$ is true, $5 \%$ of the data sets will cause the analyst to reject $H_{0}$ at the $5 \%$ significance level.

Many analysts are happy to reach this kind of incorrect conclusion only 5\% of the time. Suppose, however, that a single study tests many different hypotheses. Even if all the hypotheses are true, the probability of rejecting one or more of them is large, just from the randomness of the data. An aphorism states, "It is always possible to flog the data until it confesses."

Table 3 provided one example of testing several hypotheses. As a second example, consider hypothetical data from a single system at a number of nuclear power plants. We can test for a difference between plants, a difference between equipment manufacturers, a difference between system configurations, and so forth. (The hypothesis $H_{0}$ in each case is that no difference exists.) We can test each hypothesis for failure to start, failure to run, and perhaps for other failure modes. We can test the hypotheses using data from several distinct data sets, such as unplanned demands on the system and various types of surveillances. In addition, for each plant we can test whether the probability of failure to start is below some regulatory limit. The list of possible tests can go on and on. If any one of these tests rejects the corresponding hypothesis $H_{0}$, the naive tendency is to say "Aha, we have found something!" The wiser analyst would say "MAYBE we have found something. If we look long and hard enough, we will probably find some apparent peculiarities resulting from chance alone." 
To quantify these situations mathematically, list all the tests, and let $A_{i}$ be the event "the $i$ th test rejects the corresponding $H_{0}$ at level $p$." Then use the formulas developed next. The formulas are different for two cases:

Case 1: Independent Tests. If the tests are independent, as may happen when they are based on distinct data sets, an exact expression can be given. Denote the complement of any set $A$ by $\bar{A}$, and suppose that $n$ independent tests are performed. Then we have

$P$ (at least one test rejects at level $p$ )

$$
\begin{aligned}
& =1-P(\text { every test fails to reject at level } p) \\
& =1-P\left(\cap \bar{A}_{i}\right) \\
& =1-\Pi P\left(\bar{A}_{i}\right) \quad \text { by independence } \\
& =1-(1-p)^{n} .
\end{aligned}
$$

In the example with the counts and plant operating years from Table 3, the three tests are based on independent event counts. Therefore, the tests are independent. We then have

$P($ at least one test rejects at level 0.0093$)=1-(1-0.0093)^{3}=0.0276$.

This is small enough (compared to the usual cutoff of 0.05 ) that the earlier conclusion stands: the older and newer BWRs have different rates for equipment failure.

Case 2: Dependent Tests. When the tests are dependent, only inequalities can be given. Boole's inequality states that

$P\left(\right.$ at least one $\left.A_{i}\right) \equiv P\left(\cup A_{i}\right) \leq \Sigma P\left(A_{i}\right)$

This is closely related to the Bonferroni inequality, which states that

$P\left(\right.$ all $\left.A_{i}\right) \equiv P\left(\cap A_{i}\right) \geq 1-\Sigma\left[1-P\left(A_{i}\right)\right]$

To see the plausibility of Inequality (1), consider Figure 8, where the probability of an event $A_{i}$ is represented by the area of the corresponding circle. It is obvious that the area of the union of the circles is less than or equal to the sum of the areas of the individual circles. Note further that if the circles overlap only slightly, the inequality is almost equality. If, instead, the circles lie almost on top of each other, the inequality is far from equality.

Suppose now that $n$ tests are performed, and that the corresponding hypotheses $H_{0}$ are all true. What is the probability that at least one hypothesis will be rejected at the significance level $p$ ? By the 

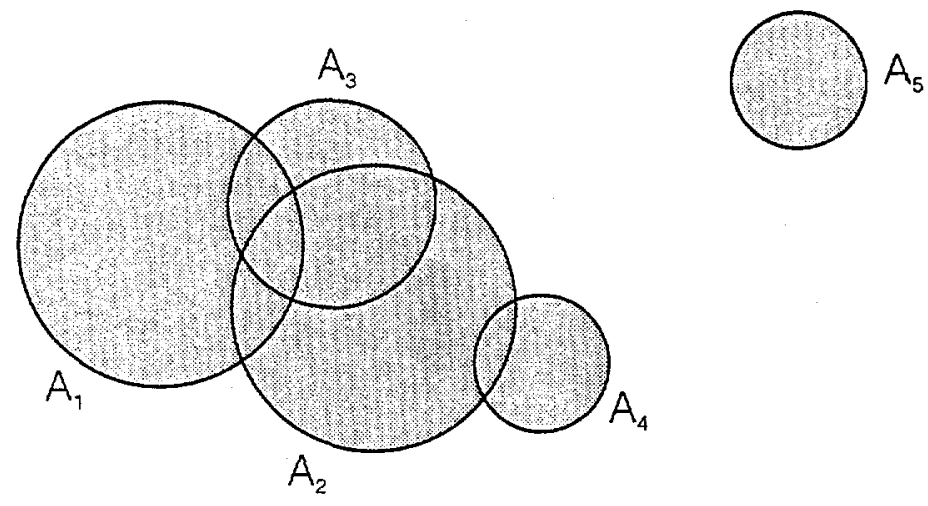

C047-WHT-495-03

Figure 8. Venn diagram with example events. Shaded region is union.

Inequality (1), it is

$P$ (at least one test rejects) $\leq \Sigma P(i$ th test rejects $)$.

The probability that the $i$ th test rejects at the significance level $p$ is just $p$, and the sum of those probabilities is $n p$. Therefore, we have

$P($ at least one test rejects at significance level $p) \leq n p$.

In the example from Table 3, we have

$P($ at least one test rejects at level 0.0093$) \leq 3 \times 0.0093=0.0279$.

This is not far from the exact value calculated above, 0.0276 .

If, instead, 30 tests were performed, not necessarily independently, and the smallest achieved significance level was 0.0093 , the probability of such an event would be

$P($ at least one test rejects at level 0.0093$) \leq 30 \times 0.0093=0.28$,

which is large enough to be within the realm of plausibility. Note, the inequality is not equality, and the overall probability has been shown to be $\leq 0.28$. 
When the tests are dependent, so that the exact probability cannot be calculated, how close to equality is the inequality? This is difficult to evaluate, but is aided by the following refinement of the Inequality (1):

$P\left(\right.$ at least one $\left.A_{i}\right) \equiv P\left(\cup A_{i}\right) \geq \Sigma_{i} P\left(A_{i}\right)-\Sigma_{i<j} P\left(A_{i} \cap A_{j}\right)$

Note, the direction of the inequality is reversed from Inequality (1). The summation on the far right is the sum of probabilities of the form $P\left(A_{i}\right.$ and $\left.A_{j}\right)$. If $n$ tests are performed in all, the sum over $i$ and $j$ has $n(n-1) / 2$ terms. Inequality (2), like Inequality (1), can be justified from consideration of diagrams like Figure 8.

In the example with counts and plant operating years from Table 3, Inequality (2) becomes

$P($ at least one test rejects at level 0.0093$) \geq n p-[n(n-1) / 2] p^{2}$

$$
\begin{aligned}
& =0.0279-0.0003 \\
& =0.0276 .
\end{aligned}
$$

This lower bound is virtually identical to the true value, 0.0276 . Suppose, instead, that 30 independent tests had been performed. The exact value would be $1-(1-p)^{n}=0.244$. Inequality (2) would say

$P($ at least one test rejects at level 0.0093$) \geq n p-[n(n-1) / 2] p^{2}$

$$
\begin{aligned}
& =0.279-0.038 \\
& =0.241,
\end{aligned}
$$

which is not far from the exact value, 0.244 .

\subsubsection{Discussion}

Several practical consequences follow from the above considerations of multiple tests.

1. Do not believe a "statistically significant" result simply because of a reported $p$-value. Instead, use the above formulas to account for the effect of the number of tests performed.

2. In applying the formulas, consider carefully how many tests to count. Think about what you know before the test is performed, and do not count unnecessary tests. An unnecessary test is one where you are very sure in advance of what the answer will be, so that the test is very unlikely to reveal information. Here are two examples: 
- If no conceivable mechanism might produce an effect, do not count the test of the hypothesis of no effect. For example, if engineering considerations say that overall plant design should have no effect on the performance of a diesel generator in the plant, do not count the test of this hypothesis.

- If the data set is so sparse that the test has virtually no power to detect an effect, do not count the test. You already know that the test will not be able to reject $H_{0}$.

In the first example, you may wish to perform the test and thereby confirm the engineering analysis, and in the second a computer procedure may automatically perform a full suite of tests even if some data sets are sparse. In neither case, however, are you performing a test as a serious attempt to discover something. Therefore, do not count them when accounting for the multiple tests.

3. If two test results are highly correlated, consider two calculations to account for the multiple tests, counting the tests as two tests and counting the tests as just one test. For example, if two plants at one location are counted as a single station, a data set might have data from 23 plants at 16 stations. Testing for a difference between plants is similar to testing for a difference between stations, and the test results will be correlated.

4. The discovery of something "statistically significant" is not the conclusion of an analysis. It is the start of an engineering analysis to determine a reason for the pattern in the data. Rely on engineering understanding, not on statistical calculations alone. Of course, this is true always, but it is especially true when the interpretation of significance levels is complicated by the presence of multiple tests. To distinguish between true departures from postulated hypotheses and apparent departures that are the result only of chance, look for mechanisms, for underlying causes. This recommendation is amplified in Section 3 below.

5. Significance levels were invented to help scientific experimenters decide whether to reject a hypothesis. A screening study differs from a focused scientific experiment, in that a large body of data is examined in many ways, perhaps resulting in a thick report embodying thousands of tests. In such a screening study, consider using significance levels (ane sny associated confidence intervals) as tools for prioritizing issues that deserve further study. Such further study might include a search for causal mechanisms and a development of data to confirm or disprove the tentative findings of the screening study.

\subsection{Consider All Sources of Variation}

This section is illustrated with three examples in which multiple sources of variation are simultaneously present. 


\subsubsection{Example: Internal Variation within Classes}

In the early 1980s, failure data for diesel generators were studied, based on Licensee Event Reports (LERs) for 1976-78. The original draft report of the study described a puzzling difference between diesel generator failures in BWRs and those in pressurized water reactors (PWRs). The failure rates were expressed as number of failures per component-hour, where a component was a diesel generator and its associated support systems, and the component-hours for a plant were the calendar hours when the plant was submitting LERs times the number of components for the plant. When the plants were categorized as PWRs or BWRs, the failure rates appeared clearly different: the chi-square test rejected the hypothesis that two reactor types had the same failure rate, and $90 \%$ confidence intervals for the two failure rates did not overlap.

This did not make sense. Why should the design of the reactor affect the performance of the diesel generators? The authors should have considered different demand rates per hour for the two reactor types, but they did not have ready access to demand data. Finally, someone pointed out that there might be variation between plants, within each reactor type. Such variation would invalidate the chisquare test, which assumes a single Poisson distribution for each of the data subsets that are compared. Accordingly, the analysts performed the appropriate tests and found clear evidence of between-plant variation, both between BWRs and between PWRs. Tolerance intervals for the failure rates at the two kinds of reactors had a large overlap-the tolerance intervals were wide because they reflected the between-plant variation. The final report (Atwood and Steverson 1982) does not differentiate between PWRs and BWRs. Instead it treats them all as plants from one population having varying failure rates, and only briefly mentions the earlier confusion.

This illustrates the need to keep in mind the assumptions of tests. For the simple chi-square tests for equality of binomial probabilities or of Poisson rates (see statistics texts, or Atwood 1994a and Engelhardt 1994), an assumption is that each class-such as BWRs and PWRs-produces data from a single binomial or Poisson distribution. The test does not have the nominal probability of Type I error if a data subset is not homogeneous, for example, if the plants in a class have differing binomial probabilities or Poisson rates.

\subsubsection{Example: Ignoring an Explanatory Variable}

This widely cited example, using categorical data, is discussed more fully by Atwood (1995) and by the references given there. A study attempted to relate infant mortality to prenatal care, based on data from two clinics. The analyses from the clinics individually and from the pooled data are given in Table 5. 
Table 5. Prenatal care versus infant mortality.

\begin{tabular}{|c|c|c|c|c|c|c|}
\hline & \multicolumn{2}{|c|}{ Clinic A } & \multicolumn{2}{|c|}{ Clinic B } & \multicolumn{2}{|c|}{ Combined } \\
\hline & \multicolumn{2}{|c|}{ Care } & \multicolumn{2}{|c|}{ Care } & \multicolumn{2}{|c|}{ Care } \\
\hline & Less & More & Less & More & Less & More \\
\hline Died & 3 & 4 & $\overline{17}$ & 2 & 20 & 6 \\
\hline Survived & 176 & 293 & 197 & 23 & 373 & 316 \\
\hline$X^{2}$ & \multicolumn{2}{|c|}{0.084} & \multicolumn{2}{|c|}{0.000} & \multicolumn{2}{|c|}{5.255} \\
\hline$p$-value & \multicolumn{2}{|c|}{1.000} & \multicolumn{2}{|c|}{1.000} & \multicolumn{2}{|c|}{0.026} \\
\hline
\end{tabular}

The $2 \times 2$ tables show the number of infants that died soon after birth or survived, according to whether they received less prenatal care or more. Beneath each $2 \times 2$ table are two numbers: $X^{2}$ is the Pearson chi-square statistic for testing independence of the two factors, and the $p$-value is the attained significance level from Fisher's exact 2-tailed test. (These are explained in many statistics books and in Atwood 1994a.) The tables for the individual clinics show virtually no relation between prenatal care and infant mortality; for example, at Clinic B the fraction $17 /(17+197)$ is almost the same as the fraction $2 /(2+23)$. However, the table with the combined data shows a statistically significant relation. In fact, the combined data give a wrong conclusion. When other things (as summarized by the variable "Clinic") are held constant, prenatal care has no evident relation to infant mortality. The apparent conclusion from the combined data does not account for the substantial differences between the two clinics, with their differences in clientele and perhaps also in equipment and procedures. In the literature of categorical data analysis, this is called "Simpson's paradox."

The paradox is partially explained by Figure 9. This figure plots the response (observed mortality, defined as number of deaths divided by the number at risk) against level of prenatal care. The size of the dot corresponds to the amount of data entering the observation; a large dot is based on more infants than a small dot. The figure shows that Clinic B has a higher response than Clinic A, and that, within each clinic, the response does not depend on level of care. The dashed line shows the response based on the pooled data; it is a weighted average of the responses from the two clinics. The number of infants with less prenatal care was about the same at the two clinics, so the left end of the dashed line is approximately halfway between the two solid lines. However, the number of infants with more prenatal care was much greater at Clinic A than a Clinic B, so the right end of the dashed line is close to the line for Clinic A. The resulting downward slope of the dashed line appears to show that more care reduces the mortality. However, this conclusion is incorrect, as shown by the level lines for each clinic. The false conclusion results from pooling data sets with different failure probabilities and unbalanced numbers of trials. 


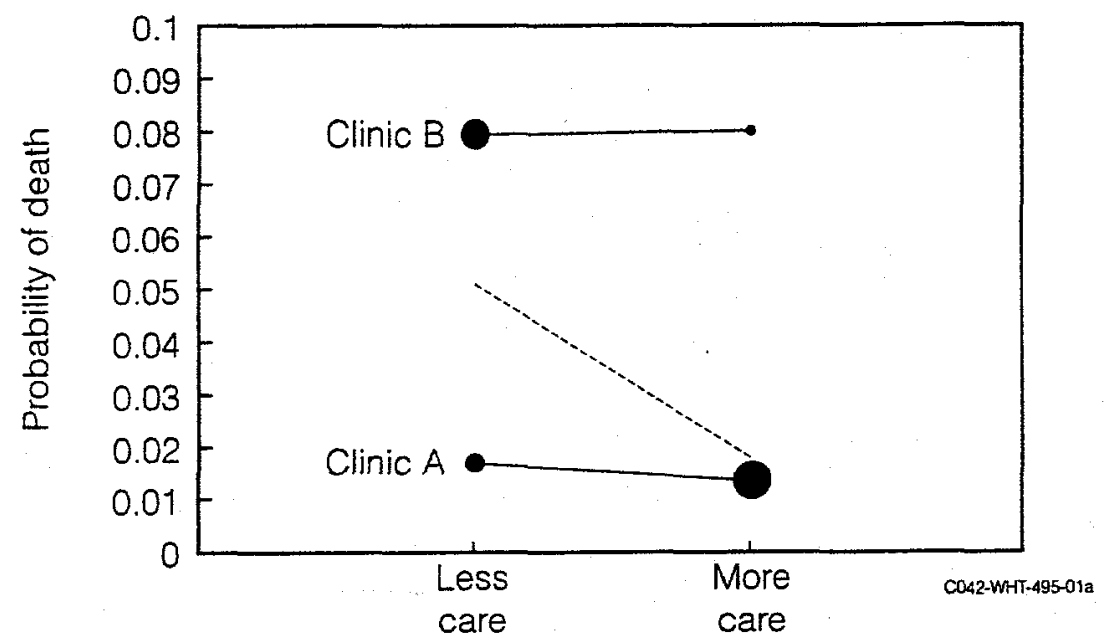

Figure 9. Observed mortality versus prenatal care and clinic. Dot size suggests amount of data. Dashed line results from (mistakenly) pooling clinics.

\subsubsection{Example: Mixing Components}

The report that produced Figures 3 and 4 also contains Figure 10, showing the age at failure of seven types of components in the RCIC system. These components are valves, valve operators, bistables/ switches, circuit breakers, transmitters, governors, and turbines. If some components, such as governors, typically do not last as long as other components, such as turbines, the graph reflects different mixtures of components at different ages on the horizontal axis. This makes such a graph extremely difficult to interpret. The report's authors therefore examine the data for each component separately.

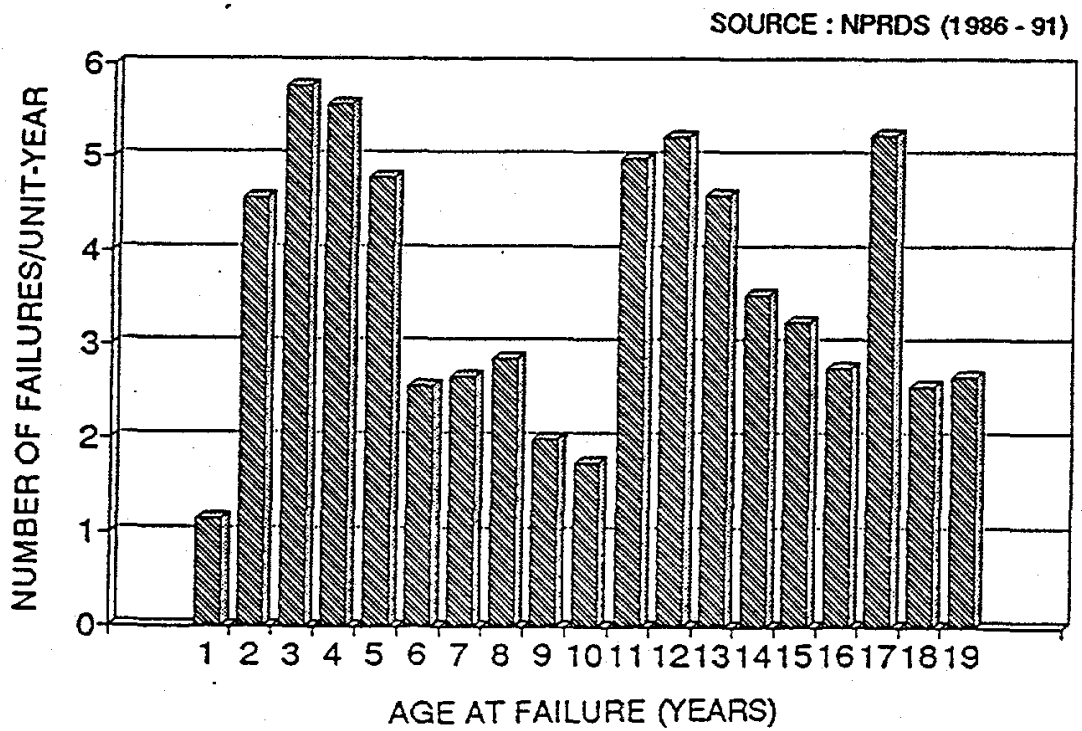

Figure 10. Combined failure frequency for seven components. Different components may dominate different portions of the graph. 


\subsubsection{Discussion}

The above examples all teach the same lesson. Data were pooled from different populationsplants in the first example, clinics in the second, components in the third. Ignoring this fact can lead to incorrect conclusions. The analyst must account for all sources of variation, either by accounting for all the sources of variation simultaneously, as in the first example or by performing separate analyses on homogeneous subsets of the data, as in the third example. The second example has aspects of both approaches.

In the first two examples considered above, a standard test was performed, based on the pooled data and ignoring the internal heterogeneity. In both cases, an unimportant difference appeared very important. Examples can also be constructed, by redrawing the lines and reweighting the dots in Figure 9, in which true differences are masked when the data are pooled. Agresti (1990) gives such an example, concerning the death penalty verdict for individuals convicted of homicide; the effect of the defendant's race reverses depending on whether or not the race of the victim is also considered. See that reference or Atwood (1995) for further discussion.

These examples demonstrate that it can be incorrect to look at pooled data one variable at a time. Such an analysis can lead to false conclusions. When several sources of variation are present, such as reactor type and plant unit in the first example or care level and clinic in the second example, the analyst should try to account for the presence of all the sources simultaneously. If this is impossible, the analyst must be aware of the issue, and either try to estimate the magnitude of its effect or partition the data into more homogeneous sets.

\subsection{Summary}

This section has illustrated errors that can be made in analyzing data. To avoid them, adhere to the following practices:

- Normalize count data correctly. First, decide on a quantity that is meaningful to study, typically a probability or rate that is directly related to the process producing the data. Then divide the count by the right denominator to estimate this quantity.

- When drawing inferences about the process that produced the data, account for the random variation in the observed values. In the presentation, show this variation by using error bars (for example, 90\% confidence intervals) or a similar device. When inferences are to be drawn, avoid bar charts for presentation, because it is hard to draw the error bars around the ends of the bars. 
- When many tests are performed in a single study, be aware that some tests will lead to rejection of the null hypothesis, just from chance alone. Do not perform unnecessary tests, and use the exact calculations for independent tests or Inequalities (1) and (2) for dependent tests to estimate or bound the true probability of finding statistics that appear significant.

- When the data contain several sources of variation, either perform separate analyses of more homogeneous subsets of the data or analyze the pooled data while considering the sources of variation simultaneously. Be aware of Simpson's paradox. Understand that looking at a pooled data set one variable at a time can lead to false conclusions. 


\section{WHAT TO DO WHEN A MODEL IS REJECTED}

\subsection{General Principles}

Suppose that a model has been hypothesized: for example, the probability of failure on demand is the same for all data sources, or the occurrence rate for some type of event is constant over time. Suppose also that a statistical test of the hypothesis has been performed, and that it rejects the hypothesis. What then?

The analyst might be inclined to postulate a more complicated model, one that includes variability between data sources or that models a time-dependent event rate. However, it is premature to do this immediately. First ask, "What is happening here? Why do the data show this departure from the simple model? What mechanisms were at work to produce the data?” A surprising pattern in the data offers an opportunity to learn more about reality, to discover new truth! A model does not explain a phenomenon, it only describes it. Therefore, do not settle for a mere superficial description of the pattern, a description expressed as a model. Instead, seize the opportunity to understand nature better! Once we understand the reality behind the data, then we can consider whether and how to model that reality.

Applied statisticians sometimes think of themselves as detectives. In that analogy, $p$-values and plots are clues, not solutions. That is, statistical tools such as tests of hypotheses and diagnostic plots are able to highlight patterns in the data. They do not find reasons for the patterns. For this, the statistician must work together with those who understand the subject matter behind the data, such as engineers, scientists, experienced technicians, or operators. The statistician's role is largely to explain in layman's language what pattern has been seen in the data, to ask probing questions, and to help assess whether various conjectures could explain the pattern. The role of the subject-matter experts is to learn what happened to generate the numbers, for example by studying the event narratives or by talking with other knowledgeable persons, and to suggest reasons for the pattern.

Once a conjecture, or "hypothesis," has been proposed to explain the observed pattern, the scientific method is to test the hypothesis. If several plausible conjectures have been suggested, they should all be tested. The original data may contain enough information to allow for a statistical test of such a hypothesis. Or new data may be needed. Indeed, explaining the pattern may require an investigation far beyond the scope of the original study that discovered the pattern.

One conjecture to be considered is that the data in the first study just happened to look weird, because of randomness alone. This is plausible if the statistical test rejected the simple model with a significant but fairly large $p$-value, say in the range from 0.01 to 0.05 . It is especially plausible if many data sets were examined in the course of the study and this data set was noticed simply because it had 
the smallest $p$-value; recall Section 2.3 on accounting for multiple tests. For example, false alarms with $p$-values of 0.01 occur one time in 100 , and can be seen when many data sets are examined. If the hypothesis that the pattern is just a fluke in the data is plausible, the way to test the hypothesis is by a confirmatory study with different data, to see if the pattern is reproducible. This may not be possible with operational data.

A related hypothesis to consider is that the data are contaminated by extraneous sources of variation. For example, event narratives can be difficult to interpret and classify. The resulting data could have extra variation, if a classification depends to some extent on the mood of the person who writes the event report or the one who later classifies the event for the database. This is especially true if the data are not peer reviewed. Many standard tests assume a binomial or Poisson distribution. When such a test is performed on data with extra variation, the probability of rejecting the null hypothesis may differ from the nominal value that the software calculates. An example of this is given below in Section 3.2.3. The analysts initially classified some events, taking care to be correct and consistent. Nevertheless, when additional information was considered some of the events were reclassified, and the apparent random variation between plants was thereby reduced.

This hypothesis may be impossible to test statistically, but the study report should describe the measures taken to prevent extraneous variance, and should state any possible reasons why the data might have more variance than modeled.

In summary, suppose that a statistical test has rejected a simple model. The steps to follow are:

1. Identify the characteristics (the pattern) in the data that caused the test to reject the model. This is done through examining the analysis output: the plots and printouts.

2. Formulate conjectures (hypotheses) to explain the causes of the data pattern. The statistician and subject-matter experts generate these hypotheses in open-ended discussions. Thorough discussions may take a long time. One of the conjectures to consider is that the pattern in the data is a false alarm, the result of randomness, in spite of the calculated significance level being less than some nominal cut-off. Another conjecture to consider is that the data are contaminated by extraneous variation, through misclassified events, unreported events, or similar causes.

3. Try to test the hypotheses generated in Step 2.

4. If

- at least one conjecture cannot be tested, and cannot be dismissed as implausible for other reasons, or 
- at least two conjectures were tested and not rejected, so that there are competing conjectures, or

- all the conjectures that could be thought of were tested and rejected,

then the results are inconclusive. In the report on the study, describe the pattern and the various explanations that were conjectured. Describe how these conjectures were tested, if they could be tested. Give any beliefs on the plausibility of the non-rejected conjectures, with reasons for these beliefs, but state that they are beliefs and opinions, not hard facts. Perhaps recommend further studies.

5. If instead

- you were very thorough in generating conjectures, and

- $\quad$ every plausible conjecture could be tested, and

- $\quad$ exactly one conjecture was not rejected, and it is supported by engineering or scientific insight,

then you apparently have discovered something previously unknown to you. If this finding would be of interest to others, consider writing an article for a meeting or a journal that deals with the subject matter behind the data. The statistician and the subject-matter experts should write the article as coauthors.

\subsection{Examples}

Several examples are discussed here to illustrate this process.

\subsubsection{Air Conditioners That Appeared to Improve with Time}

Proschan (1963) studied the air conditioning systems in 13 selected jet airplanes from a fleet of 720 planes. The data consisted of failure times. The first statistical analysis assumed an exponential time to failure, equivalent to a constant failure rate (see Engelhardt 1995b), and attempted to estimate the failure rate. However, a plot of theoretical and empirical cumulative distribution functions (c.d.f.'s) for the failure times showed that the data did not fit the assumed model very well. In particular, the two c.d.f. curves crossed only once; the empirical c.d.f. was larger than the theoretical c.d.f. for early times, and a smaller for late times. This indicates a decreasing failure rate, not the constant theoretical failure rate. The 1963 paper does not report the analysts' subsequent conjectures and tests, although it mentions that a decreasing failure rate could be the result of greater experience, debugging, or elimination of faulty parts. The article then gives two analyses to show that for each airplane no trend (or nonconstant failure rate) could be seen, and that it seems safe to assume that individually the planes have constant failure 
rates. However, the pooled data represented a mixture of different constant failure rates. Proschan then gives a theorem stating that if the individual failure rates are constant or decreasing, the failure rate of the mixture must be decreasing.

The article does not state how the fleet owner modified its maintenance policy to account for the different failure rates of the different airplanes, but the insights revealed by the analysis presumably led to different actions than if all the planes had had the same rate. This 1963 paper has become famous in reliability circles.

\subsubsection{Between-Plant Variation of Diesel Generator Failure Rates}

The example in Section 2.4.1 dealt with diesel generators at BWRs and PWRs. When the original test found a statistically significant difference between the diesel failure rates in the two kinds of reactors, the analysts sought an explanation. When between-plant variation was proposed as a cause, the analysts performed the appropriate tests and found clear evidence of between-plant variation, for each type of reactor. Tolerance intervals for the diesel generator failure rates at the two kinds of reactors had a large overlap. Many possible reasons exist for between-plant variation, including differences in hardware, environment, procedures, and personnel. Therefore, Atwood and Steverson (1982) built this plantto-plant variation into their model, and quantified it.

\subsubsection{Between-Plant Variation for HPCl Failures to Start}

An early draft predecessor to Grant et al. (1995) considered failure to start (FTS) on demand in the HPCI system. This data set is also used as an example by Atwood (1994a). Having learned from earlier studies, the analysts for the HPCI study examined the data for evidence of between-plant variation. The chi-squared test rejected the hypothesis of a common failure probability for all plants. The $p$-value was 0.001 , indicating strong evidence against the hypothesis.

Before leaping to a model to quantify this variation, the analysts considered two plausible ways that the variation could occur: there could be a few plants that are outliers, or there could be general variation that reveals itself in all plants. A plot of confidence intervals for the individual plants (Figure 4 of Atwood 1994a) shows scatter, but no single plant seems to be responsible for most of the variation. Formal hypothesis tests were based on contingency tables and chi-squared statistics: When all 23 plants were used, the $p$-value was 0.001 . When the plant that contributed most to the chi-squared statistic was dropped, the $p$-value rose to 0.004 . When the plant that contributed the next most was also dropped, the $p$-value rose to 0.030 . Only when three plants were dropped was the $p$-value greater than 0.05 (it was 0.565 ). The analysts then had a choice of (a) modeling three failure probabilities for the outliers and a fourth probability for all the other plants, using a total of four parameters, or (b) modeling general variation that affected all the plants, using two parameters. Following the principle of parsimony, 
described in Section 4.2.2, the analysts chose the second option. The variation was quantified by assuming that $p$, the probability of failure to start on demand, varies among plants, following a beta distribution. The resulting estimates are described more fully by Atwood (1994a).

It is interesting to note in retrospect that some of the variation seen in this example resulted from errors in the data classification. The data were eventually reclassified based on more information than appeared in the event narratives. The final data set, in Grant et al. (1995), still shows variation between plants, but the $p$-value is less extreme, 0.029 instead of 0.001 .

\subsubsection{Causes of RCIC Failures}

The example discussed in Section 2.1.2 in connection with Tables 2 and 3 does not exactly illustrate the process outlined in Section 3.1, because the simple model was not rejected by a statistical test. Nevertheless, the example illustrates the digging process, in which the cause of a quirk in the data was sought. The analysis of Table 2 suggested some differences between some classes of BWRs, although the differences were not statistically significant. The nature of the difference was not clear; it could have been a-difference for equipment failures, personnel errors, or both. The follow-up analysis of Table 3 found a statistically significant difference between plant groups for equipment failures. The follow-up engineering review then searched for causes of the differences.

\subsubsection{Recovery from Failures}

The draft predecessor to Grant et al. (1995) investigated the HPCI system in boiling water reactors. If the system fails to start automatically on an injection signal, the operator can attempt to recover the system by starting it manually. Similarly, if the system fails after it is running, the operator can attempt to recover the system by restarting it.

Data were collected from both unplanned actuations (true needs for the system) and periodic surveillance tests of the full system, performed once per operating cycle. Then the investigators wanted to know if these two data sources could be pooled. If the data sets are consistent with each other, pooling them is advantageous, because it creates a bigger data set to use for estimating the failure probabilities. The data from the draft study are shown in Table 6; the failure modes considered are failure to start (FTS), failure to recover from FTS (FRFTS), failure to run (FTR), and failure to recover from FTR (FRFTR).

A contingency table was constructed for each of the four failure modes, with two rows for the numbers of successes and failures, and two columns for the two data sets. Chi-squared tests were then performed. For three of the failure modes, the difference between the two data sets was not statistically

significant. For failure to recover from FTS, however, the difference was very significant, with a $p$-value 
Table 6. Failure and recovery data for HPCI.

Failure mode

Failure to start (FTS)

Failure to recover from FTS (FRFTS)

Failure to run (FTR)

Failure to recover from FTR (FRFTR)
Unplanned actuations $^{a}$

$$
6 / 57
$$

$0 / 6$

$6 / 57$

$4 / 6$
Surveillance tests ${ }^{a}$

$12 / 92$

$10 / 12$

$9 / 82$

$8 / 9$

$a$. In each column, the numerator is the number of failures and the denominator is the number of demands.

* Point estimate I s0\% Conf int

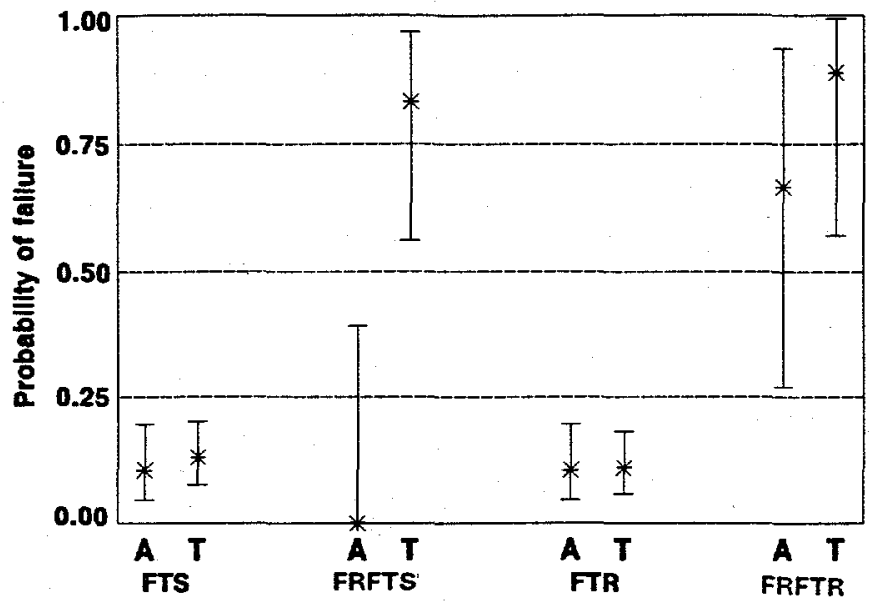

Figure 11. Probabilities of various failure modes, for unplanned actuation (A) and surveillance test (T) data sets.

of 0.001 . Confidence intervals for the failure probabilities are shown in Figure 11. Note that the unplanned actuation (A) and surveillance test $(T)$ data give very different confidence intervals for the probability of FRFTS.

With hindsight, the reason for the difference was obvious: When the HPCI system is truly needed but fails to start, the operator is strongly motivated to try to recover the system. If the system fails to start on a surveillance test, on the other hand, the natural operator action is not to try to start the system manually. Instead, the natural course of action is to record what happened, terminate the test, and try to fix the problem. With this understanding, the analysts realized that the data sets should not be pooled to estimate the probability of recovery from FTS. For recovery, only the unplanned actuations represent actual operating conditions, and only that data set should be used. 
Moreover, the same understanding applies to FRFTR, failure to recover from failure to run. For this failure mode, even on the unplanned actuations most failures were unrecovered, and therefore no significant difference could be seen between the two data sets. Nevertheless, the agreement was regarded as a coincidence, and only the unplanned actuations were used to estimated the probability of FRFTR.

The final report (Grant et al. 1995) does not even mention this data analysis. It states, "Recoveries are typically not attempted after a failure on a test. . . . Therefore, useful data for the [recovery failure modes] were found only in the unplanned demands, not in the cyclic surveillance tests." However, the authors achieved this understanding of the obvious by investigation after a preliminary hypothesis had been rejected. 


\section{BUILDING A BETTER MODEL}

Operational data are collected from a process whose primary purpose is to manufacture something, to produce electric power, or to do something else besides just generating data. Such data normally come from a variety of facilities, times, locations, or operating conditions. This gives a natural grouping of the data into subsets. When a parameter (a quantity such as $p$ or $\lambda$ ) is estimated from the data, the estimate based on a single data subset may vary among the subsets by a statistically significant amount. Such variation might not be present in a carefully designed and controlled experiment, but it is typically present in observational studies, and becomes visible when enough data are collected. Several examples have been given in Section 3.2: variation between airplanes of mean time between failure for air conditioning systems; variation between plants of diesel generator failure rates; and variation between plants of HPCI probabilities of failure to start.

The process of data analysis described in the introduction typically begins with the simplest possible model: all the data come from one homogeneous population, whose distribution depends on a single unknown parameter. When extra sources of variation are discovered, a more complicated model is required. This section considers ways to model variation between data subsets, and gives some guiding principles for deciding which of several possible models to use.

\subsection{Ways to Model Variation}

A correct data analysis will try to attribute as much of the variation as possible to specific characteristics of the data subsets, and to describe the rest as residual variation. Typically, a quantity $\theta$ is to be estimated. Examples of $\theta$ are $p$, the probability of failure on demand, and $\lambda$, the occurrence rate of some event of interest. The following are some commonly encountered ways to attribute variation in $\theta$ to characteristics of the data subsets.

(1) The quantity $\theta$ has one value for one group of data subsets, an unrelated value for another group of data subsets, and so forth, with a small number of distinct values in all. For example, a failure probability $p$ might be different for several design classes of plants. In this example, the data subsets are the plants, and the groups are the design classes. As a second instance of this type of variability, $\theta$ can take one value for all but one data subset, and be different for a single data subset, an outlier; for example, all the plants may have the same $p$, except for one outlying plant. The simplest example of this case is for $\theta$ to take the same value for all the data subsets.

(2) There are many values of $\theta$, which can be considered a sample from a continuous distribution. The distribution of $\theta$ models the unexplained variation among the data subsets. A typical example is that a failure probability, $p$, or event rate, $\lambda$, shows plant-to-plant variation. 
Case (1) is simple to implement in practice: perform a separate analysis for each group of data subsets. For example, if there are two groups of data subsets, each with a different $\theta$, do one analysis for each group.

Case (2) depends on the details of the situation. If the parameter of interest is a binomial parameter $p$, a beta distribution is a versatile and convenient distribution for modeling the variation of p. This method is explained by Atwood (1994a). Similarly, if the parameter of interest is a Poisson rate $\lambda$, a gamma distribution is a versatile and convenient distribution, as explained by Engelhardt (1994). The parameters of the beta or gamma distribution are estimated from the data. Such models are known as empirical Bayes models.

More complicated situations arise when the data subsets have more than one known relevant characteristic, such as reactor vendor, design class, plant, component manufacturer, age, and calendar time. These known characteristics are also called covariates. The two cases above generalize to the following.

(1') Each covariate can take a fairly small number of values, and each covariate individually influences the value of $\theta$. For example, $p$ or $\lambda$ is based on, say, a vendor effect and a system effect, and every data subset corresponds to some known vendor and some known system.

(2') Some or all of the covariates can take many values, and the values are thought of as coming randomly from a continuous population. For example, $p$ or $\lambda$ could depend on both the reactor vendor and the plant, with the plant effect treated as random. As another example, $p$ or $\lambda$ for a diesel generator could depend on both the diesel manufacturer and the plant. In the first example, plants are nested within vendors, and in the second example plants and manufacturer are crossed, as explained in texts on linear and loglinear models, and in Atwood (1995).

Case (1) is a special case of $\left(1^{\prime}\right)$. For example, if Case (1) involves two groups of data subsets, the covariate specifies the group, and takes two values. Case (2) is also a special case of $\left(2^{\prime}\right)$, when no covariates except the one random covariate are relevant. Finally, a third case must be mentioned.

(3) A covariate is numerical and the analysis explicitly accounts for this numerical nature. For example, $\theta$ could depend in some systematic way on time. Time could be treated as continuous, such as the exact date or exact age, or as discrete, such as calendar year. The effect of time is given by some function, such as a linear or exponential function.

A linear model can be tried, as explained by Engelhardt (1995a). However, if the fitted values of $\theta$ are illegal, such as negative estimates of a binomial $p$ or of a Poisson rate, $\lambda$, a loglinear or logit 
model can be tried, as described, for example, by Atwood (1995). More complicated models, combining features of $\left(1^{\prime}\right),\left(2^{\prime}\right)$, and (3) are also possible. The method of analysis then depends on the details.

In summary, the toolbox of a well-equipped modeler should contain these basic types of models:

- Single homogeneous population

- Homogeneous population with an outlier

- Empirical Bayes model

- Linear model

- Loglinear and logit models.

\subsection{How to Decide Which Model to Use}

Model selection is a matter of judgment and choice, not a simple mechanical procedure. This section gives several principles to guide the analyst.

\subsubsection{Understanding}

If the mechanism causing a behavior is understood or recognized, try to use a model that reflects this understanding.

The example on recoveries from failure, described in Section 3.2.5, illustrates the use of this principle. Recall that we are considering the recovery from failure, not the failure itself. There were some differences between data from two sources, unplanned actuations and periodic tests. For recoveries from FTS, the unplanned actuations showed a much higher recovery probability than did the periodic tests. For recoveries from FTR, the two data sets showed similar (small) recovery probabilities.

The analysts believed that they understood the mechanism: the operator is motivated to try to recover from failure during an unplanned actuation, and not so motivated during a test. Therefore, the probability of recovery on a test is different from the probability on an unplanned actuation. This was obvious from the data for recoveries from FTS. It was not obvious from the data for recoveries from FTR: few failures on tests were recovered, because the operator did not try; few failures on unplanned actuations were recovered, but here the nonrecoveries were presumably in spite of operator attempts to recover. The similarity of the recovery probabilities is coincidental. Based on this understanding, the analysts modeled different recovery probabilities for unplanned actuations and for periodic tests, whether or not the data displayed this difference. 


\subsubsection{Parsimony}

This principle has many guises: Occam's Razor (after William of Occam, 1280-1349), the KISS principle (an acronym for "Keep It Simple, Stupid"), the statement "Everything should be as simple as possible, but no simpler" (attributed to Einstein), and the principle of parsimony. In the present context, it says to choose the simplest model that is consistent with the data. One way to measure simplicity is by the number of parameters that must be estimated. This is the way that Box and Jenkins (1970) describe the parsimony principle: "employ the smallest possible number of parameters for adequate representation."

For example, suppose that data consist of many subsets, and that a statistical test rejects the hypothesis that $\theta$ is the same in all the data subsets. How should the variation be modeled? The principle of parsimony says to choose the simplest model of the variation. The only one-parameter model, namely a constant $\theta$, is not consistent with the data. Several two-parameter models suggest themselves: (a) a continuous distribution for $\theta$, described by two parameters; (b) a division of the data subsets into two identifiable classes, with a single value $\theta_{1}$ corresponding to the first class and a different value $\theta_{2}$ corresponding to the second class; (c) an outlier, with value $\theta_{0}$, and all the other data subsets taken together, with a common $\theta$. There are also models with more than two parameters: continuous distributions with three or more parameters, division of the data subsets into three or more classes, etc. The principle of parsimony says that if any of the two-parameter models adequately fits the data, the more complicated models should not be considered. Parsimony does not decide which of the two-parameter models to select, unless the analyst or customer thinks that one of them "seems simpler" than the others.

\subsubsection{Economy of Presentation}

This is Tukey's (1961) version of the principle of parsimony: "It may pay not to try to describe in the analysis the complexities that are really present in the situation." Like the previous principle, it is based on a desire for simplicity. Now, however, we seek not a simple explanation of the facts, but a simple presentation.

The following example is taken from Atwood (1983), based on an analysis by Meachum and Atwood (1983). Failure rates (denoted $\lambda$, with units $1 / \mathrm{hr}$ ) were found for five applications of pressure switches in commercial nuclear power plants of the BWR design. The five applications were reactor pressure switches, containment pressure switches, condenser low vacuum pressure switches, turbine control fast closure valve pressure switches, and turbine first stage pressure switches. Following Meachum and Atwood, these are called systems of switches here. Within each system, statistically significant between-plant variation was found. The variation between plants within each system was modeled by a continuous distribution for $\lambda$, a gamma distribution. For example, 24 plants had reactor 
pressure switches, so the gamma distribution for this system was estimated from 24 observations. The means and $90 \%$ intervals of the five gamma distributions are shown in Figure 12.

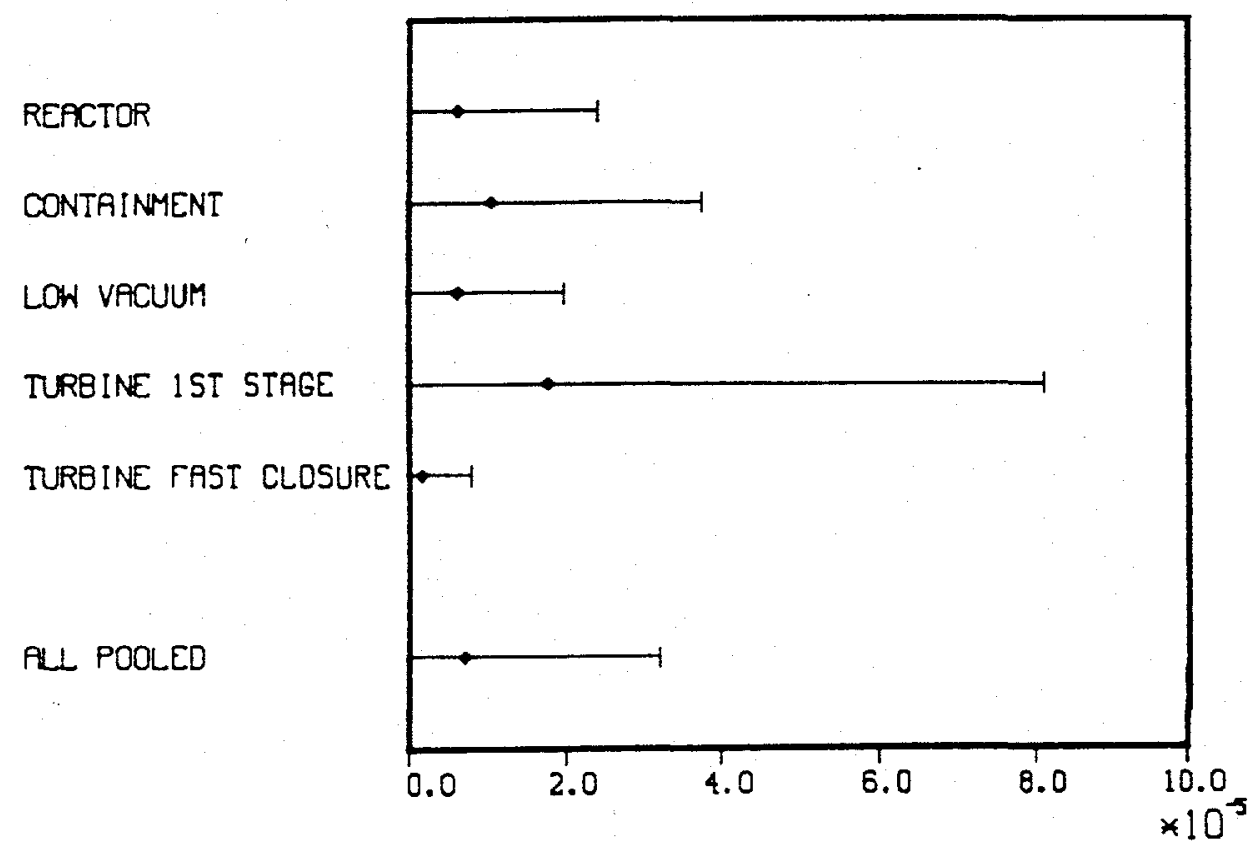

Figure 12. Failure rates for pressure switches-Bayes means and $90 \%$ intervals.

In addition, the data for pressure switches in all the systems were analyzed together, with between-plant and between-system variation modeled by a single gamma distribution. This distribution was based on 24 observations from the reactor pressure switches at 24 plants, plus 33 observations from the containment pressure switches at 33 plants, plus the observations from the other systems of pressure switches. The mean and $90 \%$ interval for this distribution are shown at the bottom of Figure 12 .

Now the analysts asked which analysis should be used, the one yielding five intervals or the one yielding one interval. The question was not whether the distribution of $\lambda$ was exactly the same for all five systems. (That question could have been addressed using the likelihood ratio test-see Section 4.2.4.) Some variation between systems is inevitable and is accounted for, along with variation between plants, in the distribution based on the pooled data. The real question is whether there is any advantage in treating the systems separately. That is, are the estimates more precise for the separate systems than for the pooled systems? A made-up example is shown in Figure 13, with components from three systems.

In Figure 13, information is lost by pooling the systems. The intervals for the separate systems are all much shorter than the pooled interval, so keeping the systems separate allows more precise estimates. Figure 12, on the other hand, gives little reason for treating the systems separately. The 


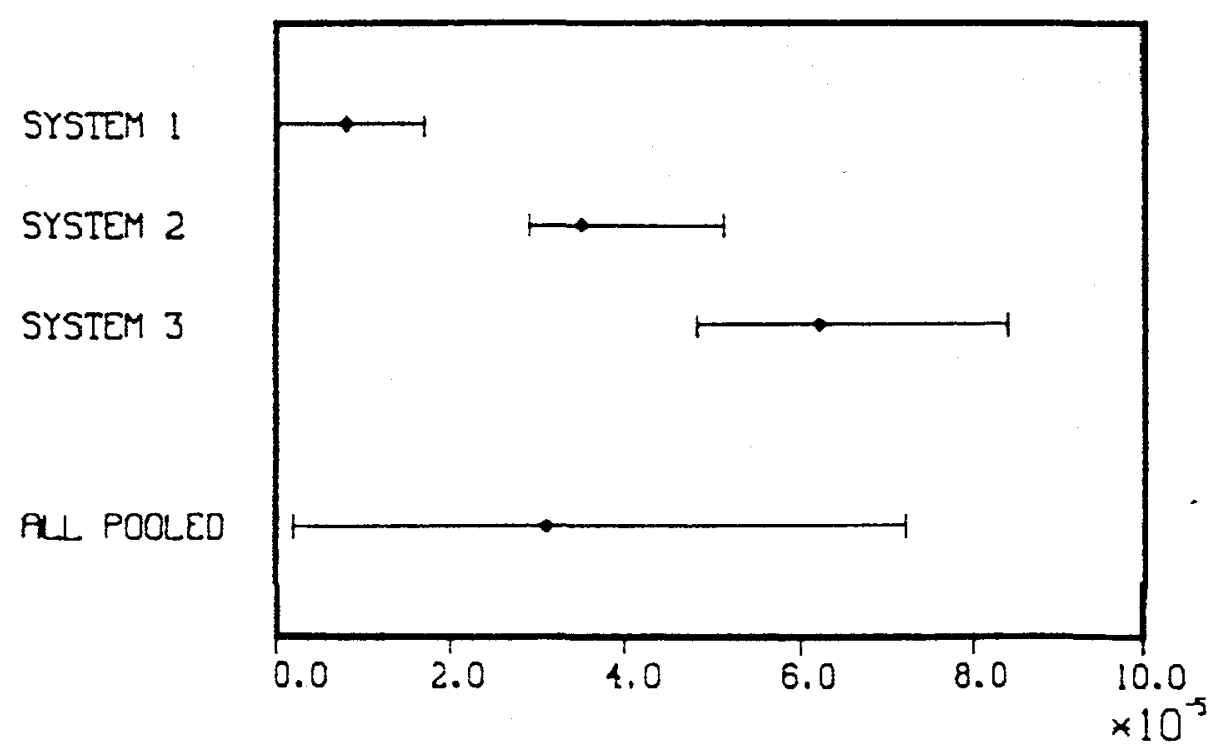

Figure 13. Hypothetical failure rates-Bayes means and $90 \%$ intervals.

turbine fast closure pressure switches have a short interval; if those switches are of special interest, they could well be analyzed separately. But in the actual data analysis, the desire for simple answers led the authors to treat all the pressure switches together. The model choice was based on the principle of economy of display.

\subsubsection{Goodness of Fit}

Goodness of fit was considered above only qualitatively: the principle of parsimony said not to use a complicated model if a simple model fit the data adequately. Now we use goodness of fit in a more quantitative way.

The principle is to try to choose the model that best fits the data. This can be restated as: choose the model that accounts for the largest portion of the variance, and therefore reduces the residual variance the most. The exact method of measuring the goodness of fit depends on the context. The principle, however, is used in many settings of statistical model building. For example, in the context of linear models (or multiple regression), standard methods try to maximize the correlation between the response variable and the explanatory variables, thus minimizing the residual variance. Similarly, loglinear model selection is often based on choosing the model that maximizes the likelihood of the data, subject to constraints on the complexity of the model.

The principle of parsimony calls for simplicity, while goodness of fit calls for complexity. Therefore, linear modelers may use measures such as adjusted $R^{2}$, Mallows' $C_{P}$, or the Akaike information criterion to balance the advantages of using more or fewer parameters. These refinements are described in some texts on linear models, but are not considered here. 
When a loglinear model is used, as described by Atwood (1995) and the references given there, parameters may be added sequentially to produce a chain of nested models. Each model in the chain is a generalization of the simpler models and a special case of the more complex models. Each model can be tested against the alternative of the next more complex model, either by the likelihood ratio test or by a version of the Pearson chi-square test. Based on judgment and the test results, the analyst chooses a model that fits adequately and whose fit cannot be greatly improved by going to the next step in complexity. Several examples are given by Atwood (1995). They are too long to repeat here but they should be read. One example is given here, which is similar to those except that neither model is a special case of the other.

Suppose that we are trying to model a situation where there may be a single outlier. In practice, such examples seem to be unusual. More typically, if one outlier is removed another outlier pops up. Nevertheless, suppose that we wish to consider the possibility of a single outlier. To be specific, suppose the parameter of interest is $p$, the probability of failure on demand. The data consist of a count of demands, $d_{j}$, and of failures, $f_{j}$, at the $j$ th plant, where $1 \leq j \leq J$, with $J$ defined as the number of plants. Treat $d_{j}$ as fixed and $f_{j}$ as random at each plant. Call the possible outlier Plant 1 . The two models considered are:

Model $D$. The parameter $p$ has a discrete distribution, with a value $p_{1}$ for $p$ at Plant 1 and a different value $p_{0}$ for all the other plants. The model's name is mnemonic for "discrete." At Plant $1, f_{1}$ is binomial $\left(d_{1}, p_{1}\right)$, and at any other $\operatorname{Plant} j, f_{j}$ is binomial $\left(d_{j}, p_{0}\right)$.

Model $B$. The parameter $p$ follows a beta $(\alpha, \beta)$ distribution. The model's name is mnemonic for "beta." At any particular plant, once $p$ has been selected from this distribution for the $j$ th plant, $f_{j}$ is binomial $\left(d_{j}, p\right)$. This is an empirical Bayes model.

The models are qualitatively different, and it is not obvious how to decide which model fits the data better. The principle of parsimony offers no help, because both models use the same number of parameters, $p_{1}$ and $p_{0}$ for Model D, and $\alpha$ and $\beta$ for Model B. The following method, therefore, is based on goodness of fit.

The two models both yield a discrete distribution for $f_{j}$, binomial under Model D and betabinomial under Model B. Under both models, the failure counts $f_{j}$ at different plants are independent of each other. Therefore, both models for the distribution of the failure counts are special cases of the full model:

Model $F$. The parameter $p$ has a value $p_{j}$ at Plant $j$. The model's name is mnemonic for "full." At Plant $j, f_{j}$ is binomial $\left(d_{j}, p_{j}\right)$. 
Model F specifies no particular relation among the $p_{j}$ values, but special cases such as Models $\mathrm{D}$ and $\mathrm{B}$ arise when the $p_{j}$ values are related. The likelihood ratio test can be used to compare either Model D or Model B with Model F. For example, to see if Model D must be rejected in favor of Model $F$, look at the likelihood ratio test statistic

$G^{2}($ Model D | Model F $)=-2 \log \Lambda_{\mathrm{DF}}$

where

$\Lambda_{\mathrm{DF}} \equiv$ (likelihood of data under Model D, with $p_{1}$ and $p_{0}$ estimated by MLEs) /

(likelihood of data under Model F, with $p_{j} s$ estimated by MLEs) .

Here, MLE stands for maximum likelihood estimate. The quantity $G^{2}$ is calculated by many statistical packages; for example, SAS PROC GENMOD (1993) can handle Model D, and it prints $G^{2}$ as the "likelihood ratio chi-square" statistic. When the sample size is large, the quantity $G^{2} \equiv-2 \log \Lambda_{\mathrm{DF}}$ has approximately a $\chi^{2}(J-2)$ distribution if Model D is correct, and a larger distribution otherwise. Therefore, the test gives a crude decision on whether Model D should be used at all.

Similarly, $G^{2}$ (Model B | Model F), defined using Model B with $\alpha$ and $\beta$ estimated by their MLEs, can be used to test whether Model B should be used at all. Users of SAS, however, must program the calculation of this test statistic, because Model B is not part of a standard SAS procedure.

The pair of tests can tell more, however, than whether either model is adequate. Together, they give a ranking of Models $\mathrm{D}$ and $\mathrm{B}$. Think of $G^{2}$ (Model D | Model F) as a measure of the distance between the Model D and Model F. A small value means that Model D fits the data almost as well as the full model, that there is very little difference between them. A large value means that Model D does not fit the data nearly as well as the full model. A comparison of $G^{2}$ (Model D | Model F) and $G^{2}$ (Model B | Model F) shows which model fits better. The better fitting model is the model with the smaller test statistic, or equivalently, with the larger likelihood.

The statistic $G^{2}$ is sometimes supplemented by a Pearson chi-square test, as discussed by Atwood (1995) and by textbooks on loglinear models. This is not always possible, however. For example, Model B does not have a natural chi-square test. Therefore, chi-square tests are not considered here.

This example is not worked out with data here, because, as mentioned above, real examples with only a single outlier are difficult to find. Working through such an example, however, would involve nothing more than calculating likelihoods, and comparing them to see which model had the larger likelihood. To facilitate this, and to make it possible to compare more complicated models that might 
arise in actual practice, formulas are given in Appendix B for some common distributions involving event counts.

\subsubsection{Feasibility}

Sometimes a model is used because any alternative model is just too hard to postulate or to construct. This is not exemplary data analysis, but if we are forced to do it, that fact must be stated clearly. 


\section{CHOOSING AND COMBINING FAILURE MODES}

This section applies to reliability estimation, when various failure modes are of interest. In a study of a kind of pump, for example, both failure to start and failure to run may be of interest, because the two kinds of failures are modeled separately in a probabilistic risk assessment (PRA). In a study of pressure relief valves, failure to open and failure to close may warrant separate study, because the two failure modes have different consequences.

Bayesian methods provide by far the easiest way to combine failure modes. Therefore, only Bayesian methods are considered in this section, both when choosing and when combining failure modes.

\subsection{Choosing Failure Modes to Model}

The failure modes to be modeled should be only those dictated by the purposes of the study. The fundamental principle is not to estimate any more quantities than necessary. The intuitive idea behind this principle is, "Do not spread the data too thin." It is yet another occurrence of the principle of parsimony. Several instances of the principle are given here, followed by a case study for illustration.

\subsubsection{Subdivide Failure Modes Only as Necessary}

The failure modes should not be subdivided more than necessary. For example, suppose that a study of pumps is only interested in failure of the pump to start and operate for the required time, and that the reported demands in the data set required the pumps both to start and to run. Suppose also that the data do not show unusual complications, such as atypical failures to start at one plant. Then the analyst should not partition the observed failures into failures to start and failures to run. Instead, every failure should be classified simply as failure to operate. Sometimes a study is interested both in overall failure to operate and in the individual failure modes. When the data are sparse, it may be advantageous to base the overall estimate on the undivided data, even though the data are broken into failure modes in a different part of the study report. The loss of simplicity in presentation may be balanced by a gain in quality of the estimate.

The effect of subdividing failure modes is now examined, first for failures on demand and then for failures in time.

Failures on Demand. Consider $f$ failures on $d$ demands, and let $p$ be the probability of failure on demand. The Bayes estimator $p^{*}$ turns out to have the form

$p^{*}=(1-w) \hat{p}+w \mu$ 
where $\hat{p}$ is the MLE $f l d, \mu$ is the prior mean, and $w$ is a weighting factor. This is seen as follows. The prior distribution on $p$ is beta $(\alpha, \beta)$, and the posterior distribution is beta $(f+\alpha, d-f+\beta)$. The Bayes point estimator $p^{*}$ is the mean of the posterior distribution,

$$
\begin{aligned}
p^{*} & =(f+\alpha) /(d+\alpha+\beta) \\
& =f /(d+\alpha+\beta)+\alpha /(d+\alpha+\beta) \\
& =[d /(d+\alpha+\beta)][f / d]+[(\alpha+\beta) /(d+\alpha+\beta)][\alpha /(\alpha+\beta)] \\
& =(1-w) \hat{p}+w \mu
\end{aligned}
$$

where $\hat{p}$ is the MLE $f / d, \mu$ is the prior mean $\alpha /(\alpha+\beta)$, and $w=(\alpha+\beta) /(d+\alpha+\beta)$.

That is, the Bayes point estimator is a weighted average of the MLE and the prior mean. The MLE is pulled toward $\mu$ by $w$. The MLE is unbiased, but the Bayes estimator is biased towards $\mu$. The presence of a bias is not in itself bad, as discussed below, but its existence must be kept in mind. The Jeffreys noninformative prior distribution has $\alpha=\beta=1 / 2$, so when this noninformative prior is used we have $\mu=0.5$ and $w=1 /(d+1)$.

To apply this to the above example with failure to start and failure to run, let $p_{S}$ and $p_{R}$ denote the probabilities of the two failure modes, and let $f_{S}$ and $f_{R}$ denote the number of failures of each mode. Let $p$ denote the probability that a pump fails to operate, that is, it fails to start or fails to run. This probability is approximately the sum $p_{s}+p_{R}$, if both failure probabilities are small. By pooling the data, with $f=f_{S}+f_{R}$ failures to operate in $d$ demands, we have the overall Bayes estimator of $p$, given by Equation (3).

Now consider estimating the two failure probabilities separately and then adding the estimates to obtain an estimate of $p$. If the pump fails to start, there is no demand for it to run, but for this example we will take $d>f_{s}$, so that the number of demands to run as nearly equal to the number of demands to start, $d$. Therefore $w$ is treated as the same for both failure modes. The point estimator is

$$
\begin{aligned}
p_{s}^{*}+p_{R}^{*} & =(1-w) \hat{p}_{s}+w \mu+(1-w) \hat{p}_{R}+w \mu \\
& =(1-w)\left(\hat{p}_{s}+\hat{p}_{R}\right)+2 w \mu \\
& =(1-w) \hat{p}+w \cdot 2 \mu
\end{aligned}
$$

The estimate is a weighted average of $\hat{p}$ and $2 \mu$, not of $\hat{p}$ and $\mu$. The resulting bias is greater than when the failure modes were combined. Some bias is desirable: the MLE is unbiased, but it equals 0 when there are no observed failures, and so the MLE is unrealistic in that case. But it is also unrealistic to divide the failures into many unnecessary modes and obtain the same bias for each mode. That makes the overall bias too large. 
The above discussion gives considerations for the analyst, not laws in stone. If the number of demands is large, $w$ is small and the absolute bias is small whether it involves $\mu$ or $2 \mu$. If $\hat{p}$ is not tiny, the relative bias may be small. When both the absolute and the relative biases are small, that is, when the data set is fairly large, subdividing the failure modes may be useful. In any case, the analyst must consider the costs and benefits of dividing or combining failure modes.

Failures in Time. Now consider a different setting, in which failures occur randomly in time instead of on demand. Based on $n$ failures in time $t$, we wish to estimate the failure rate $\lambda$. The MLE is $n / t$, and the Bayes estimate from the noninformative prior distribution is $(n+0.5) / t$. This cannot be interpreted as pulling the MLE, $n / t$, toward the prior mean, because the so-called noninformative prior distribution is an improper distribution and the prior mean does not exist. Nevertheless, the Bayes estimator is biased by $0.5 / t$, and if several such estimates (for example, corresponding to different failure modes that could randomly occur independently of each other) were added, the biases would be added. As with failures on demand, subdividing failure modes has the cost of additional bias.

\subsubsection{Try to Avoid Estimating Failure Probabilities when No Failures Occurred}

The case when no failures are observed $(f=0)$ in $d$ demands is an extreme example. If $d$ is quite small, the bias of the Bayes estimator is very large, and this large bias is completely an artifact of the small number of demands and the chosen prior distribution. As $d$ increases the bias decreases, but until a failure is observed we do not know if the bias has decreased most of the way or only a small part of the way. It is quite possible for the estimate to be orders of magnitude too large. This happens when far too few demands are observed to allow any appreciable chance of a failure occurring. The opposite is not true, however; if very many demands are observed, so that at least one failure almost certainly occurs, the estimated probability of failure will be moderately close to the true value, not orders of magnitude too small.

Therefore, be especially cautious when asked to estimate a failure probability based on no observed failures; instead, combine failure modes where possible to avoid this situation. If estimation based on no observed failures cannot be avoided, state that the failure probability can be bounded on the high side reasonably well, but without additional information, the probability cannot be bounded on the low side, except by zero.

\subsubsection{Do Not Estimate Quantities for Which No Data Exist}

The previous example had limited data: 0 failures in $d$ demands. Sometimes, however, data do not exist at all. For example, the data narratives may not specify whether motor-operated valves failed to open or failed to close. In such a case, data do not exist for the specific failure modes, and the only failure mode that should be modeled is failure to operate. As another example, the study may be 
interested in a pump's failure to start automatically on a signal, and, if it fails to start automatically, whether the operator could recover the pump and make it start somehow. If the data record no failures to start automatically, there were no opportunities for the operator to recover the pump, and no data for estimating the probability of failure to recover. In such a case, failure to recover should not be modeled as a separate failure mode.

It is mathematically possible to model the failure to recover, even with no data-the posterior distribution is the same as the prior distribution. However, it is not smart to do something just because it is possible. At best, this use of the prior distribution can be called modeling, not estimation or data analysis. A model based on a noninformative prior and no data does not necessarily yield realistic conclusions.

An analysis report should clearly state the effect of omitting a failure mode. Consider failure to recover, for example. This typically occurs as an input to an AND gate, such as (failure to start AND failure to recover). Omission of this failure mode is mathematically equivalent to setting $P$ (failure to recover $=1$, which is conservative. As a different example, suppose we postulate the failure mode "failure to run after long operation," for example because of eventual cumulative stress. Suppose that this failure mode is not observed because all the demands in the data set were of short duration. The failure mode would typically occur as an input to an OR gate, such as (failure to start OR failure to run after a short time OR failure to run after long operation). Omitting the failure mode is mathematically equivalent to setting $P$ (failure to run after long operation) $=0$, which is anticonservative. In this case, the report should clarify that the data only allow estimates for demands of short duration.

\subsubsection{Split Data into Subsets Only as Necessary}

The above examples of splitting and combining failure modes are similar in many ways to another kind of example: If the probability of failure seems to be the same in different data subsets, such as different years, different facilities, or different types of demand, the pooled data should be used to estimate a single failure probability; separate probabilities for each data subset should not be used, except perhaps to show that they all appear similar. This kind of example does not combine failure modes-the definition of failure is the same for each data subset. Instead, it pools the data subsets used to estimate the failure probability.

The principle of parsimony, explained in Section 4.2.2, calls for simple models. As a consequence, data should be split into subsets only when engineering understanding or statistical evidence says that they have different parameter values. Sometimes, however, data must be split nevertheless. For example, the customer may wish to see annual reliabilities, even though the data show no evidence of changes in time. We now consider the effect of splitting data unnecessarily, and mention a way to avoid the worst consequences. 
First, we perform calculations similar to Equation (3), but now split the data by year. Let $f_{i}$ and $d_{i}$ denote the number of failures and demands in the $i$ th year, let $f$ equal $\Sigma f_{i}$, and let $d$ equal $\Sigma d_{i}$. Let $p$ be the probability of failure on demand, the same for every year. For any one year, the weighting factor $w_{i}$ is $\delta /\left(d_{i}+\delta\right)$, where $\delta=\alpha+\beta$ for the prior distribution, and for the pooled data the weighting factor $w$ is $\delta /(d+\delta)$. Each $w_{i}$ is larger than $w$, because each $d_{i}$ is smaller than $d$. Therefore, each year's MLE, $\hat{p}_{i}$, is pulled further toward $\mu$ than the overall MLE $\hat{p}$ is. The average of the $\hat{p}_{i}$ values is pulled toward $\mu$ roughly by the average of the $w_{i}$, and so is pulled more than $\hat{p}$ is. Therefore, less overall bias results from pooling the data over the years and estimating $p$ than by estimating $p$ for each year and averaging the estimates.

For example, suppose that Equation (3) is applied to 7 years of data, with $d_{i}=10$ demands in the $i$ th year, and suppose that the Jeffreys noninformative prior is used, so that $\alpha=\beta=1 / 2$ and the prior mean, $\mu$, is $\mathbf{0 . 5}$. Then the Bayes estimator for the $i$ th year is

$p_{i}^{*}=\left[d_{i} /\left(d_{i}+1\right)\right] \cdot \hat{p}_{i}+\left[1 /\left(d_{i}+1\right)\right] \cdot 0.5=(10 / 11) \hat{p}_{i}+(1 / 11) \cdot 0.5$

Each yearly MLE is pulled toward 0.5 by $1 / 11$. On the other hand, the overall MLE, with year combined, is pulled toward 0.5 by $1 /\left(\Sigma d_{i}+1\right)=1 / 71$.

If it is necessary to divide the data, for example by year, the above problem of bias can be mitigated by using a prior mean that is consistent with the data. Atwood (1994b) defines constrained noninformative prior distributions as having a specified mean but being otherwise uninformative, in a sense made precise. The constrained noninformative prior for a Poisson rate $\lambda$ is shown to be a gamma distribution with the specified mean and with a shape parameter $1 / 2$. The constrained noninformative prior for a binomial parameter $p$ is approximated by a beta distribution with the specified mean. When the mean is less than 0.5 , the first parameter of the beta distribution is somewhat smaller than 0.5 -values are tabulated by Atwood (1994b).

The suggested implementation of constrained noninformative prior distributions is as follows. First pool the data and use the Jeffreys noninformative prior to obtain an overall mean. For binomial data with a total of $f$ failures and $d$ demands, the overall mean for $p$ is $(f+1 / 2) /(d+1)$. For Poisson data with a total of $f$ events in time $t$, the overall mean for $\lambda$ is $(f+1 / 2) / t$. Let the prior distribution be the constrained noninformative prior with this mean. Then split the data into subsets. For example, split the data into data from various years. For each subset, use the data for that subset to update the prior. As always, the MLE for each data subset is pulled toward the prior mean, but now the prior mean is the overall mean from the pooled data. In many applications, this is more acceptable than pulling all the MLEs toward some other prior mean such as 1/2. An example in Section 5.3.2 will illustrate this point. 
In summary, model only the failure modes required by the study, especially if some modes have no observed failures, and model failure modes only if the data allow their estimation. This is illustrated here by a case study.

\subsubsection{Case Study: HPCS System}

Eight BWRs have a high pressure core spray (HPCS) system. Roesener et al. (1995) examine 7 years' data from this system. The system was challenged by unplanned demands and by surveillance tests performed once per plant operating cycle. However, the cyclic tests did not test the injection valve under actual operating conditions. Therefore the HPCS system was broken into the "bulk of the system," that is, the entire system except for the injection valve, and the valve. In 72 demands (30 unplanned demands and 42 cyclic surveillance tests) the bulk of the system never failed. In 24 of the unplanned demands, the injection valve was also demanded to open, and it never failed. Both types of postulated failure were of interest.

For calculating unreliability, note that

$P($ failure to start)

$=P($ bulk of system fails to start or injection valve fails to open)

$\doteq P$ (bulk of system fails to start) $+P$ (injection valve fails to open) .

The Bayes estimate of a probability of failure, based on the Jeffreys noninformative prior, adds $1 / 2$ to the number of failures and 1 to the number of demands. Therefore the approximate estimate of the probability of failure to start, based on the last line of Equation (5), was

$[(0$ failures of bulk of system $)+0.5] /[(72$ demands on bulk of system $)+1]$

$+[(0$ failures of injection valve $)+0.5] /[(24$ demands on injection valve $)+1]$

$=0.5 / 73+0.5 / 25$

$=0.027$.

Alternatively, following the advice of Section 5.1.2 to combine failure modes having no observed failures, one could base an estimate only on the demands that challenged the entire system, including the injection valve. Then the estimated probability is

$[(0$ failures of entire system $)+0.5] /[(24$ demands on entire system $)+1]=0.5 / 25=0.020$.

The value in Equation (7), $0.5 / 25$, equals a portion of the value in Equation (6), although the events counted are somewhat different in principle. 
Recall the remark in Section 5.1.2, that when no failures are observed, drastic overestimation of the true failure probability is quite possible, but drastic underestimation is extremely unlikely. The fact that Equation (7) gives a smaller value than Equation (6) suggests that the small size of the data set, coupled with the Jeffreys prior, may be artificially inflating the estimate in Equation (6). Therefore, in an intermediate draft, Roesener et al. used Equation (7) when calculating the unreliability, ignoring a portion of the data to minimize the number of times that the Jeffreys prior could contribute its bias. However, the customer felt that this was confusing, and requested that the two terms in Equation (6) be modeled separately. Because the numerical difference in the answers was small compared to the overall uncertainty, the report authors agreed to this request.

When the authors proposed using $0.5 / 25$, based on only the unplanned demands, they also considered going further, combining failure to start with failure to run and updating the prior distribution only once with a single data set. However, the unplanned demands almost never challenged the system's ability to run; most run times were short and, furthermore, the transfer of suction sources was never challenged by an unplanned demand. Therefore, the probability of failure to run was estimated from the cyclic surveillance tests only; this data set was disjoint from the proposed data set for failure to start. Because no single data set challenged the entire system for both failure to start and failure to run, the idea of combining the two failure modes was discarded. Instead, the contribution to unreliability of failure to start and of failure to run was estimated by

$P$ (failure to start or failure to run)

$\doteq P$ (failure to start) $+P$ (failure to run) ,

with the two parts estimated separately in both the early and revised versions of the analysis.

Because there were no failures on unplanned demands, there were no opportunities to recover from failure, and recovery initially was not modeled. This is conservative, because failure to recover would enter the overall unreliability through an AND gate. The customer, however, wanted to see the same failure modes, to the extent possible, in the analysis of systems such as HPCI, HPCS, and RCIC. The HPCI and RCIC systems had enough data that failure to recover could be modeled realistically. Therefore, failure to recover was modeled for the HPCS system, even in cases where no data existed. The authors consented to this for two reasons: (a) The Jeffreys prior, with mean $1 / 2$, was felt to be not too unrealistic for failure to recover. (b) The unreliability calculations that modeled recovery were primarily used for studies of possible trends in time. Modeling failure to recover affected every calculation in the same way, and therefore did not distort conclusions about trends.

In summary, various failure modes were considered. For the calculation of unreliability they were initially combined as much as possible, to minimize the bias from the prior distribution. However, the customer felt that this was hard to present, and therefore directed that the customary failure modes 
all be modeled. This example illustrates the admonitions given earlier in Section 5.1 not to attempt to estimate failure modes for which no data exist, and not to divide failure modes unnecessarily, especially if some modes have no observed failures. It also illustrates the difficulty in presenting such ideas.

\subsection{Combining Failure Modes}

\subsubsection{Formulas Using Moments, for Probabilities of Failures on Demand}

Equation (5) is an example of combining failure modes. It is an approximation, valid when the failure probabilities are small. We consider here the exact formulas for combining failure modes, and also combinations other than by "or". We also consider the uncertainty distributions of the combinations. The most practical way to obtain the uncertainty distribution for a complicated combination of failure modes is by Monte Carlo simulation. However, for relatively simple combinations, algebraic calculations can produce very good approximations. These algebraic formulas are given here. The ideas were given in print by Martz and Waller (1990) and some of the references cited there, and presumably by earlier authors as well.

Use the following terminology. Let event $A_{i}$ have probability $p_{i}$. For example, $A_{1}$ could be out of service for testing or maintenance, $A_{2}$ could be failure to start, $A_{3}$ could be failure to recover from failure to start, and so forth. Let $p_{i}$ have an uncertainty distribution with mean $\mu_{i}$ and variance $\sigma_{i}^{2}$. Let $A_{C}$ denote an event formed by combining the $A_{i} s$, and let $p_{C}$ be the probability of $A_{C}$. Denote the mean and variance of $p_{C}$ by $\mu_{c}$ and $\sigma_{c}{ }^{2}$. We further refine this notation: let $A_{i}$ and $A_{j}$ denote events whose probabilities are estimated independently; let $A_{i 1}, A_{i 2}$, etc., denote events of the same type, such as independent failures of identical components or identical subsystems, and assume that the probabilities of these events are all estimated from the same data set, so that all the estimated failure probabilities are identical. Then the formulas in Table 7 hold. The names of some of the SAS (1988) macros (andgate, notgate, orgate) follow PRA terminology. These macros are given in Appendix A.

The moment formulas of Table 7 are exact, and are derived directly from the definitions of mean and variance. To approximate the distribution of $p_{c}$, one can assume that the distribution of $p_{c}$ is of a certain form, and determine the parameters of that distribution from the mean and variance. The resulting distribution determines approximate percentiles, such as the 5th percentile, median, or 95th percentile. The approach used here requires a distributional approximation, because a mean and variance alone are not enough to determine percentiles. The approximation is good if the assumed form of the distribution is close to correct. The accuracy is discussed below for one example.

The most useful application is when $p_{c}$ is assumed to have a beta distribution. The relevant moments of a beta $(\alpha, \beta)$ distribution are given in terms of the parameters by 
Table 7. Formulas for combinations of basic events.

\begin{tabular}{|c|c|c|c|c|}
\hline$A_{c}^{a}$ & $p_{c}$ & $\mu_{C}$ & $\sigma_{c}^{2}$ & SAS macro ${ }^{b}$ \\
\hline$A_{i}$ and $A_{j}$ & $p_{i} p_{j}$ & $\mu_{i} \mu_{j}$ & $\sigma_{i}^{2} \sigma_{j}^{2}+\mu_{i}^{2} \sigma_{j}^{2}+\mu_{j}^{2} \sigma_{i}^{2}$ & andgate \\
\hline $\operatorname{not} A_{i}$ & $1-p_{i}$ & $1-\mu_{i}$ & $\sigma_{i}^{2}$ & notgate \\
\hline $\begin{array}{l}A_{i} \text { or } A_{j} \equiv \\
\operatorname{not}\left[\left(\operatorname{not} A_{i}\right) \text { and }\left(\operatorname{not} A_{j}\right)\right]\end{array}$ & $1-\left(1-p_{i}\right)\left(1-p_{i}\right)$ & $1-\left(1-\mu_{i}\right)\left(1-\mu_{j}\right)$ & $\sigma_{i}^{2} \sigma_{i}^{2}+\mu_{i}^{2} \sigma_{i}^{2}+\mu_{i}^{2} \sigma_{i}^{2}$ & orgate \\
\hline$A_{i 1}$ and $\ldots$ and $A_{i k}$ & $p_{i}^{k}$ & $E\left(p_{i}^{k}\right)$ & $E\left(p_{i}^{2 k}\right)-E^{2}\left(p_{i}^{k}\right)$ & $\begin{array}{l}\text { abtopow, for } \\
\text { beta distr. }\end{array}$ \\
\hline
\end{tabular}

a. The formulas for $p_{c}$ make the natural assumptions about the events, $A_{i}$, contributing to $A_{c}$ : if one event follows the other in time (e.g. failure to run after successful start, or failure to recover after initial failure) the failure probability of the second is the conditional probability given the first event; if the events can occur simultaneously (such as failure of two components in parallel) they are independent. The moments of the uncertainty distribution of $p_{C}$ assume that the uncertainty distributions for the $p_{i} s$ are independent. This applies to all cases in which $A_{C}$ involves more than one event $A_{i}$.

$b$. These macros are all printed in Appendix A. 
$\mu=\alpha /(\alpha+\beta)$

$\sigma^{2}=\mu(1-\mu) /(\alpha+\beta+1)$

$E\left(p^{k}\right)=[\alpha(\alpha+1) \cdots(\alpha+k-1)] /[(\alpha+\beta)(\alpha+\beta+1) \cdots(\alpha+\beta+k-1)]$

By using an intermediate term $\delta \equiv \alpha+\beta$, the above equations for the mean and variance can be inverted to give $\alpha$ and $\beta$ :

$\delta=\mu(1-\mu) / \sigma^{2}-1$

$\alpha=\mu \delta$

$\beta=\delta-\alpha$.

Macros for doing all these calculations in SAS are given in Appendix A. The moments of a $\operatorname{beta}(\alpha, \beta)$ distribution are found from the parameters by the macro abtomom, and the parameters $\alpha$ and $\beta$ are found from the moments by the macro momtoab. The mean and variance of $P\left(A_{i}\right.$ and $\left.A_{j}\right), P($ not $\left.A_{i}\right)$, and $P\left(A_{i}\right.$ or $\left.A_{j}\right)$ are evaluated in terms of the mean and variance of $P\left(A_{i}\right)$ and $P\left(A_{j}\right)$, and therefore are valid regardless of the distributions of these probabilities-no particular distribution such as beta is required for the three macros andgate, notgate, and orgate. The mean and variance involving an arbitrary number, $k$, of identical terms are found under the assumption that $P\left(A_{i}\right)$ has a beta distribution, so that Equation (8) holds.

The accuracy of the approximation was investigated for portions of the example of Section 5.3.2 below. Let $p_{c}$ now denote the probability of system failure, given by Expression (9) in that section. A 90\% uncertainty interval was found in two ways: (a) Monte Carlo simulation and (b) approximation of the distribution of $p_{c}$ with a beta distribution. Three such pairs of calculations were performed, each based on one set of prior distributions and data. In each case, the two methods gave very similar intervals. For example, the year 1993 provided a very small data set, with the most important failure mode having only two demands, and no failures. When this data set was used with the Jeffreys prior, Monte Carlo simulation with 99,000 runs produced $(0.03599,0.6848)$ as a $90 \%$ interval, and the beta approximation produced $(0.03148,0.6767)$. The upper end points differ by 0.0081 , which only is $1.3 \%$ of the length of the interval. The results were similar or better when the same data set was used with a different prior, and when the full data set ( $\geq 63$ demands for each of the two dominant failure modes) was used with the Jeffreys prior.

\subsubsection{Formulas Using Moments, for Rates of Events in Time}

The preceding section considered failures on demand. This section considers failures or other events in time, with occurrence rates having units $1 /$ time. Complicated combinations of occurrence rates are less common in practice than combinations of failure probabilities. Therefore, only a sketch is given here, paralleling some of the ideas given above for failure probabilities. 
Suppose two kinds of events can occur randomly in time, with occurrence rates $\lambda_{1}$ and $\lambda_{2}$. The rate at which either the first or the second kind of event occurs is $\lambda \equiv \lambda_{1}+\lambda_{2}$. Suppose that $\lambda_{1}$ and $\lambda_{2}$ are estimated from unrelated data sets, and that a Bayesian distribution for $\lambda$ is to be found.

Use a Bayesian update of the Jeffreys noninformative prior to estimate $\lambda_{i}$ from $n_{i}$ events in time $t_{i}$. Then the posterior distribution for $\lambda_{i}$ is a gamma distribution with mean $\left(n_{i}+0.5\right) / t_{i}$ and variance $\left(n_{i}\right.$ $+0.5) / t_{i}^{2}$. The mean and variance of the sum $\lambda_{1}+\lambda_{2}$ are the sums of the means and the variances:

$E(\lambda)=\left(n_{1}+0.5\right) / t_{1}+\left(n_{2}+0.5\right) / t_{2}$

$\operatorname{var}(\lambda)=\left(n_{1}+0.5\right) / t_{1}^{2}+\left(n_{2}+0.5\right) / t_{2}^{2}$

These calculations are exact. To obtain an approximate interval estimate for $\lambda$, treat $\lambda$ as if it had a $\operatorname{gamma}(\alpha, \beta)$ distribution. The parameters are as given by Engelhardt (1994), with $\beta$ having the same units as $t$. The values of $\alpha$ and $\beta$ are determined by the mean and variance. That is $E(\lambda)=\alpha / \beta$ and $\operatorname{var}(\lambda)=\alpha / \beta^{2}$. Thus,

$\alpha=[E(\lambda)]^{2} / \operatorname{var}(\lambda)$ and

$\beta=E(\lambda) / \operatorname{var}(\lambda)$.

Percentiles of the resulting gamma distribution can be used to approximate the uncertainty in the combined rate.

\subsection{Case Studies}

The formulas from Table 7 for binomial data are now illustrated by applying them in two cases. The first example is a typical auxiliary feedwater (AFW) system in a PWR, and the second is an application to the HPCI system by year.

\subsubsection{The AFW System}

The postulated AFW system has one turbine-driven train and two identical motor-driven trains. The system fails if all three trains fail. Different analysts may model the system in various ways. In particular, the treatment of recoveries and of common-cause failures may vary from one analysis to another, for valid reasons. Therefore, the analysis here is only an example of how such an analysis could be carried out.

The modeled failure modes for any one train are: 
MOOS Out of service for testing or maintenance

FTS Failure to start

FRFTS Failure to recover from FTS

FTR Failure to run, assuming that it has successfully started

FRFTR Failure to recover from FTR.

In addition, common cause failures are modeled:

CCFM Common cause failure (to start and run) of both motor-driven trains

FRCCFM Failure to recover from CCFM

CCFA Common cause failure (to start and run) of all three trains

FRCCFA Failure to recover from CCFA.

Finally, it should be noted that only one train is allowed to be out of service for maintenance at any one time.

To apply the formulas of Table 7, first consider individual failure of one train. (The term "individual" is used here to mean "not by common cause.") Let $p_{\text {Moos }}$ denote the probability that the train is out of service for maintenance, and assume that $p_{\text {MOos }}$ has a beta $\left(a_{\text {MOos }}, b_{\text {Moos }}\right)$ distribution. In fact, the turbine-driven train probably has a different MOOS probability from a motor-driven train. Therefore, we will eventually need a distribution for $p_{\mathrm{h}}$ moos and one for $p_{\mathrm{m}}$, moos, with subscripts $t$ and $m$ denoting "turbine" and "motor." However, for now suppress the extra subscript and consider only $p_{\text {Moos. }}$ Its beta distribution could be obtained based on an assumed prior distribution, updated by the observed number of times that such trains were out of service for maintenance (MOOS) in the observed number of unplanned demands on them. If the noninformative prior distribution is used and updated with $f_{\text {Moos }}$ MOOS events in $d_{\text {Moos }}$ demands, the posterior beta parameters are $a_{\text {Moos }}=f_{\text {Moos }}+1 / 2$ and $b_{\text {Moos }}$ $=d_{\text {Moos }}-f_{\text {Moos }}+1 / 2$. Define similar notation for the probability and the beta parameters for the other failure modes.

The train fails to perform its mission if it is out of service for maintenance, or it fails to start and this failure is not recovered, or it fails to run and this failure is not recovered. This is illustrated in Figure 14. That is, the train unreliability is

$P$ (train fails to perform its mission)

$=P[M O O S$ or (FTS and FRFTS) or (FTR and FRFTR)] .

A SAS macro, train, is given in Table 8, to evaluate train unreliability. It invokes the more primitive macros given in Appendix $A$ and listed in Table 7. For example, to use the macro for the turbine-driven train, in a SAS DATA step, define the SAS variables atmoOs for $a_{\mathrm{h}, \mathrm{moos}}$, and so forth. 


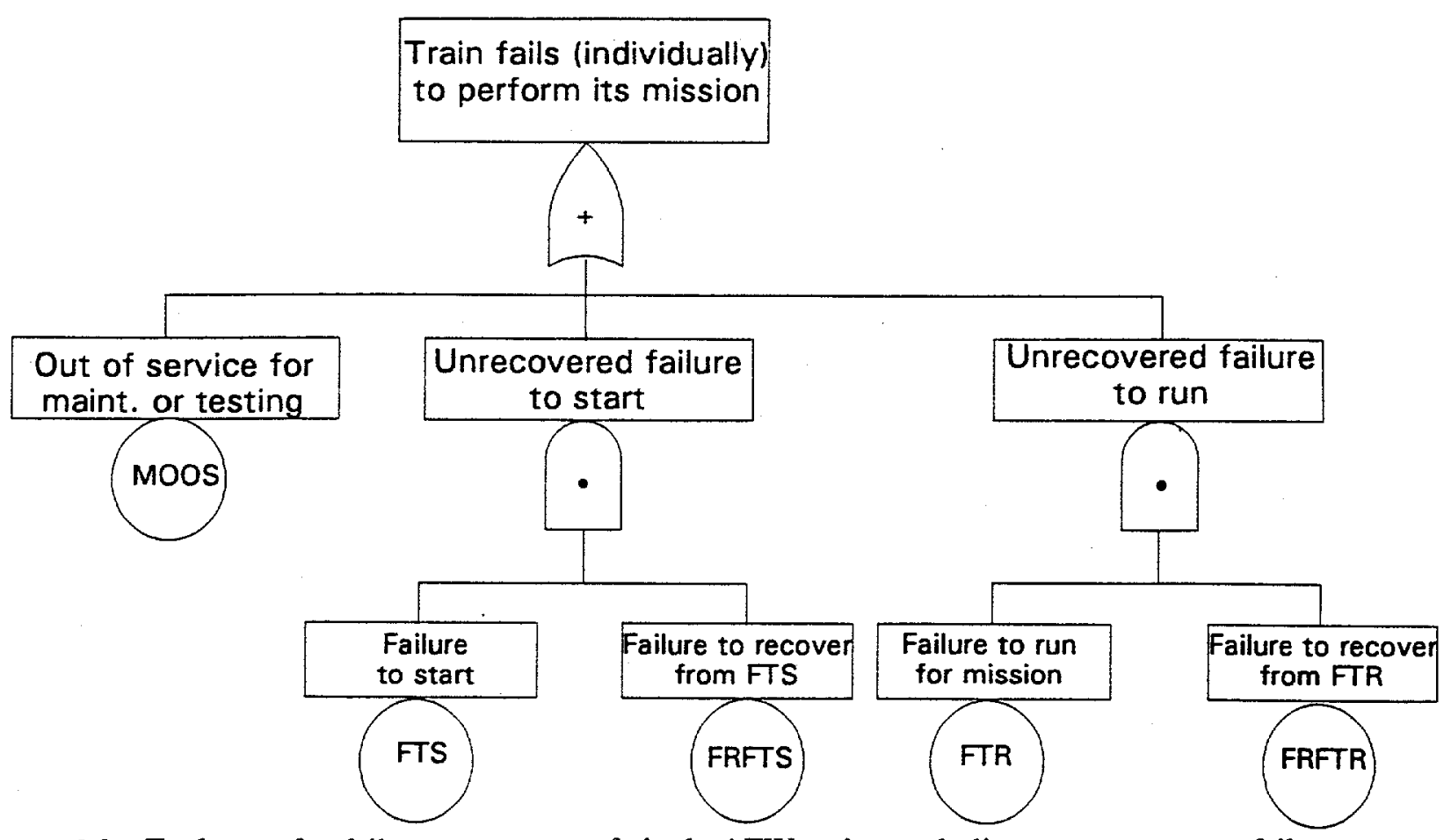

Figure 14. Fault tree for failure to operate of single AFW train, excluding common-cause failures.

Table 8. SAS macro for train unreliability, ignoring common-cause failures.

\&macrotrain (aMOOS, bMOOS, aFTS, bFTS, aFRFTS, bFRFTS, aFTR, bFTR, aFRFTR, bFRFTR, mutrain, vartrain, atrain, btrain);

* Get the moments from the parameters, assuming beta distributions; \%abtomom (\&aMOOS, \&bMoOS, muMOOS, varMoOS)

\%abtomom (\&aFTS, \&bFTS, muFTS, varFTS)

\%abtomom ( \&aFRFTS, \&bFRFTS, \&MUFRFTS, \&VFRFTS)

\%abtomom ( \&aFTR, \&bFTR, \&muFTR, \&varFTR)

\%abtomom (\&aFRFTR, \&bFRFTR, \&MUFRFTR, \&VFRFTR)

* Combine the pairs of failure modes connected by "and";

\%andgate (muFTS, varFTS, muFRFTS, vFRFTS, muFS, varFS)

\%andgate (muFTR, varFTR, muFRFTR, VFRFTR, muFR, varFR)

* Combine the three failure blocks connected by "or";

\%orgate (muMoos, varMoos, muFs, varFs, mul, var1)

\%orgate (mu1, var1, muFR, varFR, \&mutrain, \&vartrain)

* mutrain and vartrain are the mean and variance of the train unreliability;

* Now approximate the distribution by a beta, and find its parameters;

\%momtoab (\&mutrain, \&vartrain, \&atrain, \&btrain)

\%mend train; 
Having defined numerical values for these values, enter the line

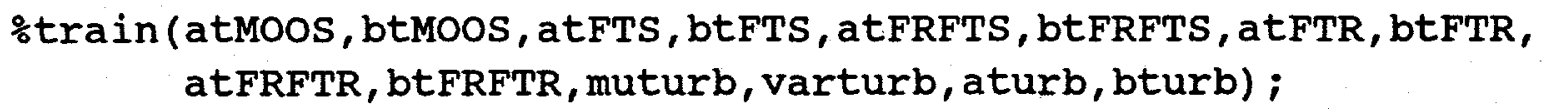

A single motor-driven train could be considered in the same way, by invoking train with arguments amMOOS, etc. This yields the exact moments of the unreliability of the train, considering only individual failures of that train. The final step of the macro tra in obtains the beta parameters, based on a beta distribution as an approximation for the true distribution of the unreliability. Such a step is necessary for Bayes intervals for the train unreliability. Monte Carlo simulation (very many replications) is the only way to obtain exact intervals. The beta approximation given here is very good, however, in a simple example such as this one, as discussed at the end of Section 5.2.1. The advantage of the above algebraic method is that it is very fast. For example, if plant-specific calculations are desired for many plants, use of the above approximation can be sufficiently accurate, and easier than doing a Monte Carlo simulation for each plant.

Having considered the unreliability of a single train, let us now consider the unreliability of the pair of motor-driven trains. The unreliability of the pair of motor-driven trains is

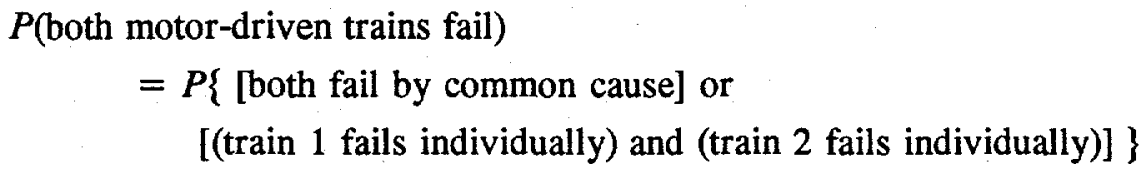

This is illustrated by the lower portion of Figure 15, and is evaluated by the SAS code in Table 9 .

The coding in Table 9 assumes that the parameters such as amMOOS have already been determined. Those determinations should be inserted in a SAS data step. Note that the individual failures of the two motor-driven trains are not combined by the macro andgate. This is because the same data set was used to estimate the failure probabilities for train 1 and train 2 . Therefore, the Bayesian distributions for the two failure probabilities are not independent, as assumed for the first line of Table 7. Instead, the two failure probabilities are identical, and so obey the fourth line of Table 7.

Note also that this method ignores the constraint that the two trains cannot be simultaneously out of service for maintenance. Because the algebra allows the possibility of both being out of service, the algebraic calculation is conservative. Unless maintenance unavailability is a major contributor to the unreliability, the conservatism is small. 


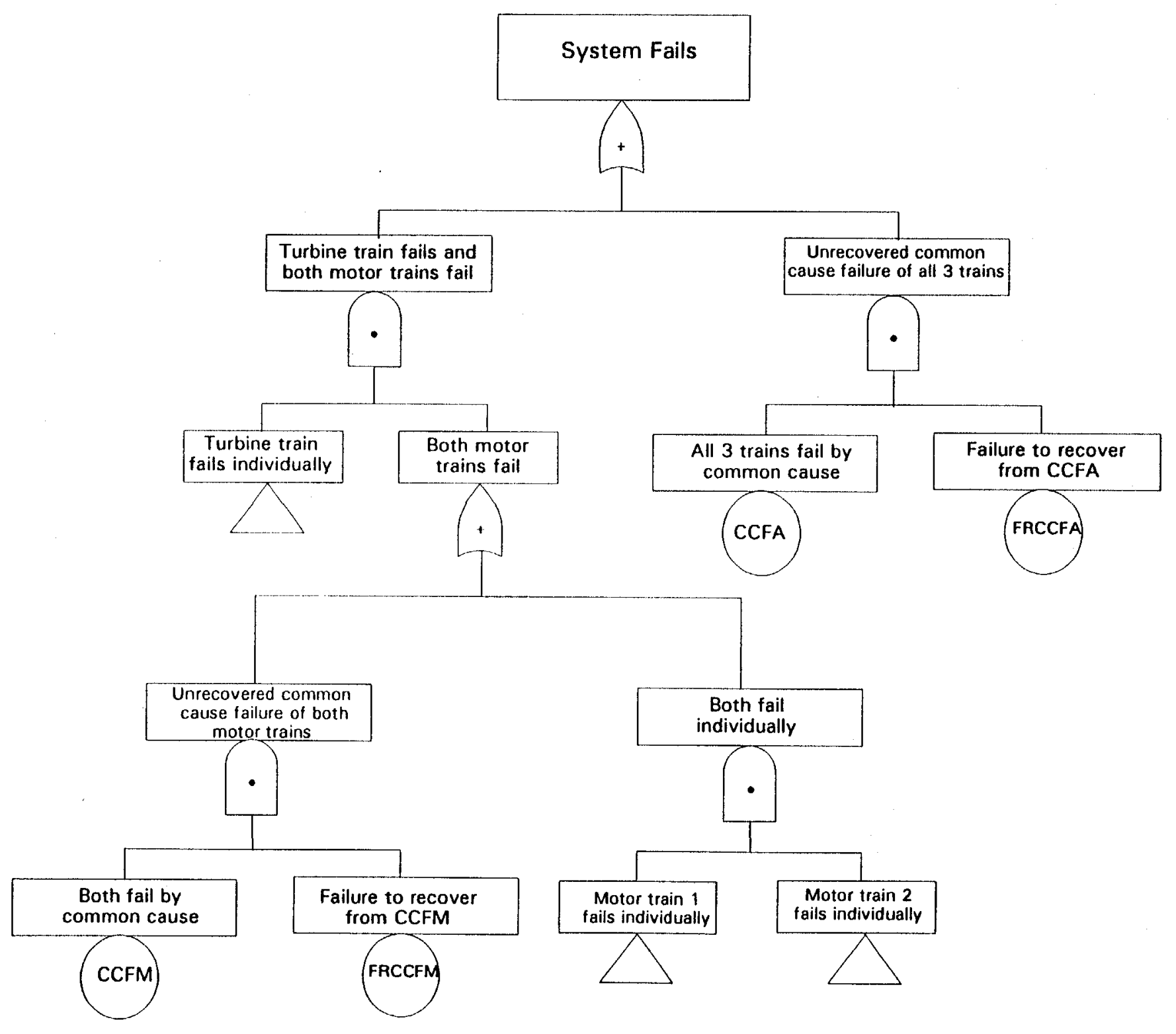

Figure 15. Unreliability of a typical AFW system. 
Table 9. SAS code for the probability of both motor-driven trains failing.

* Find the moments corresponding to one motor-driven train; \%train (amMOos, bmMOOS, amFTS, bmFTS, amFRFTS, bMFRFTS, amFTR, bmFTR, amFRFTR, bmFRFTR, mumotor, varmotor, amotor, bmotor)

* Find distribution for both trains failing individually;

* amotor and bmotor were just found, and are now used;

* The argument 2 means that the probability for one train is squared;

\%abtopow (2, amotor, bmotor, mu2mot, var2mot)

* Find the moments for the common cause probabilities;

$\%$ abtomom (a CCFM, bCCFM, muCCFM, varCCFM)

\%abtomom (aFRCCFM, bFRCCFM, MUFRCCFM, vrFRCCFM)

* Combine them by "and";

\%andgate (muCCFM, varCCFM, muFRCCFM, vrFRCCFM, muCCM, varCCM)

* Combine this with the individual part;

\%orgate (muCCM, varCCM, mu2mot, var2mot, mumot, varmot) ;

* Get the beta parameters;

\%momtoab (mumot, varmot, amot, bmot)

Also, this calculation assumes that an operator will try to recover both trains-if the first train cannot be recovered, the operator will still try to recover the second train. This assumption may be optimistic.

Finally, let us approximate the total unreliability of the three-train AFW system. This is

$P($ system fails $)$

$=P($ all three trains fail $)$

$=P\{$ [all fail by common cause] or

[(turbine train fails individually) and

(both motor trains fail) ] \}

and is illustrated by Figure 15.

To evaluate this, append the SAS code in Table 10 to that in Table 9.

Again, the constraint on being simultaneously out of service for maintenance is ignored, resulting in a small conservatism. 
Table 10. SAS code for AFW system unreliability.

* Combine the individual turbine part with the two motor trains;

* This uses the moments that were found earlier;

\%andgate (muturb, varturb, mumot, varmot, mumoturb, vmoturb)

* Find the moments for the overall common cause probability and its recovery;

$\because$ abtomom (aCCFA, bCCFA, muCCFA, varCCFA)

\%abtomom (aFRCCFA, bFRCCFA, MUFRCCFA, vrFRCCFA)

* Combine them by "and";

\%andgate (muCCFA, varCCFA, muFRCCFA, vrFRCCFA, muCCA, varCCA)

* Combine this with the individual part;

\%orgate (muCCA, varCCA, mumoturb, vmoturb, muAFW, varAFW)

* Get the beta parameters;

\%momtoab (muAFW, varAFW, aAFW, bAFW)

\subsubsection{Use of Constrained Noninformative Prior When Data Are Split into Subsets}

This example is from Grant et al. (1995), and is discussed more fully by Atwood (1994b). The HPCI system had six failure modes: (1) out of service for testing or maintenance (MOOS), (2) failure to start for reasons other than injection valve failure (FTSO), (3) failure to start because the injection valve failed to open (FTSV), (4) failure to recover from failure to start (FRFTS), (5) failure to run as long as needed (FTR), and (6) failure to recover from failure to run (FRFTR). Failure of the system when demanded equals

MOOS or [(FTSO or FTSV) and FRFTS] or (FTR and FRFTR)

and the unreliability is the probability of system failure on demand. Except for the splitting of failure to start into two modes, this is the same logical structure as shown in Figure 14.

Data were collected from unplanned demands and cyclic surveillance tests for 1987-1993. In all, there were 63 unplanned demands and 108 tests, and the unplanned demands and the tests challenged various parts of the system, corresponding to various failure modes. The chi-square goodness-of-fit test found no significant difference between years for any failure mode. Confirming this, it was not possible to fit a nondegenerate empirical Bayes distribution, modeling variation between years, for any failure mode.

Therefore, the most parsimonious estimate of system unreliability pooled the data from the 7 years, for each failure mode. For each failure mode, the Jeffreys noninformative prior was updated with the total count of failures and demands for that mode. The resulting posterior distributions were 
combined using the moment formulas given in Table 7. The resulting mean system unreliability was 0.058 , and an approximate $90 \%$ Bayes interval was $(0.024,0.103)$. (As discussed in Section 5.2.1, the approximation from using a beta distribution for the system failure probability was very good in this example.) This is shown at the right in Figure 16. The dotted horizontal line marks the mean system unreliability, 0.058 .

However, the customer wanted to see if the unreliability varied from year to year. Although no significant variation between years was seen for any one failure mode, it was conceivable that a trend or pattern might emerge when the failure modes were combined. Therefore, the unreliability was calculated for each year.

For a first attempt at a calculation, the year-specific unreliability was found in the same way as the overall unreliability, by updating the Jeffreys prior with the year's data for each failure mode, and combining the posterior distributions for the failure modes. The results are given in Figure 16, with the open circles showing means and the bars showing $90 \%$ intervals. Observe that each year's calculated unreliability is larger than the overall unreliability. The reason is that, for each failure mode, the Jeffreys noninformative prior pulls the probability toward the prior mean, 0.5 .

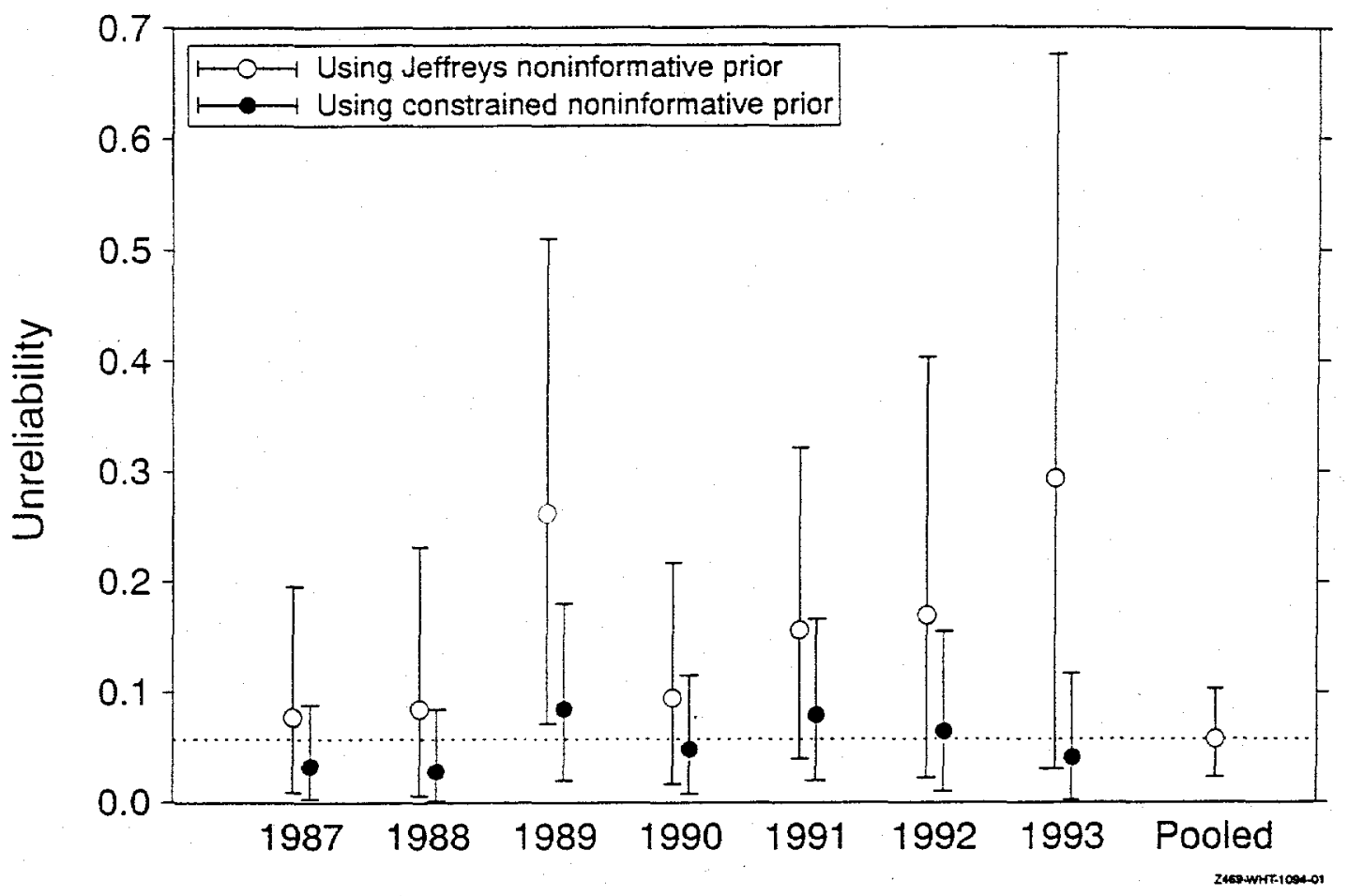

Figure 16. Posterior means and $90 \%$ intervals for unreliability, using the Jeffreys noninformative prior and the constrained noninformative priors. 
Therefore, a constrained noninformative prior distribution was used for each failure mode. For example, FRFTS had an overall total of 0 failures in 5 demands. The posterior mean, obtained by updating the Jeffreys prior with the pooled data, had a mean of $(0+1 / 2) /(5+1)=0.0833$. The constrained noninformative prior was a diffuse distribution with this mean, well approximated by a beta(0.3603, 3.963) distribution. This prior distribution was updated with each year's FRFTS data to give the year-specific posterior distribution for P(FRFTS). The other failure modes were treated similarly, and the distributions were combined using the formulas from Table 7. The resulting calculated unreliabilities are given in Figure 16, with the solid circles showing the means and bars showing $90 \%$ intervals. The means are comparable to the unreliability from the pooled data, and the uncertainty intervals are, in general, larger than the uncertainty interval when the data are pooled. These results, not those using the Jeffreys prior, were used by Grant et al. (1995).

In this example, the entire data set was used to determine the prior distribution for a portion of the data. To that extent, this example resembles an empirical Bayes analysis. However in this example only the mean is estimated from the full data set; the variance is chosen to be noninformative. In an empirical Bayes analysis, both the mean and variance would be estimated from the data. In either analysis, the data set from a single year is used twice, once to help determine the prior, and once to update the prior and give the posterior for that year. The fact that seven years contribute to the total dilutes the effect of this double use. 


\section{REVIEW}

This section reviews the recommendations made in this report. The points are developed more fully where they are introduced. This section simply contains brief reminders.

\subsection{Data Analysis for Evaluating an Explicit or Implicit Model}

- Normalize count data correctly. Divide the count by the right denominator to estimate a meaningful quantity, typically a quantity directly related to the process producing the data.

- When drawing inferences, account for random variation in the data. In the presentation, show confidence intervals or similar indicators of uncertainty.

- Be aware of the effect of performing many simultaneous tests, and try to account for it.

- Be aware of Simpson's paradox, and the dangers of looking at the explanatory variables one at a time. Try to consider the sources of variation simultaneously, or split the data into more homogeneous subsets.

\subsection{What to Do When a Model Is Rejected}

- Identify the features of the data that caused the model to be rejected.

- Discuss the issue with experts on the subject matter-engineers, scientists, technicians-and formulate hypotheses about what was truly happening. Two explanations for consideration are that the apparent pattern is a random fluke and that the data contain extraneous variance. Other hypotheses would reflect more substantive conjectures.

- Try to test these hypotheses, or at least give reasons for believing or disbelieving them.

- $\quad$ Report the results of these investigations.

\subsection{Building a Better Model}

- $\quad$ Be acquainted with standard types of models:

- Homogeneous populations following standard probability distributions 
- Homogeneous populations with outliers

- $\quad$ Empirical Bayes models

- Linear models

- Loglinear and logit models

- Use the following principles when choosing a model:

- Understanding: try to reflect the true natural process.

- Parsimony: use the simplest model that is adequate.

- Economy of display: do not present more than is useful.

- Goodness of fit: be sure that the model adequately fits the data, and among equally parsimonious models prefer the model that fits better

- Feasibility: if the selected model has deficiencies, but is the best that you can construct, say so.

\subsection{Choosing and Combining Failure Modes}

- When choosing failure modes to model,

- Subdivide failure modes only as necessary. Such a division increases the bias of the estimator.

- Try to avoid estimating probabilities when the data are sparse; if possible, combine the failure mode with another. Resist modeling a quantity for which no data exist; state that you cannot estimate the quantity.

- Split data into subsets only if necessary, that is, only if the different subsets correspond to different parameter values. If the customer requires you to split the data anyway, use a constrained noninformative prior in the analysis, not the Jeffreys noninformative prior.

- When combining failure modes, such as when computing unreliability, use Monte Carlo simulation from the posterior distributions, or algebraic formulas for the moments. 


\section{REFERENCES}

Agresti, Alan, 1983, "Fallacies, Statistical," in Encyclopedia of Statistical Sciences, Vol. 3, S. Kotz and N. L. Johnson, editors, New York: John Wiley \& Sons, pp. 24-28.

Agresti, Alan, 1990, Categorical Data Analysis, New York: John Wiley \& Sons, Section 5.2.2.

Atwood, C. L., and J. A. Steverson, 1982, Common Cause Fault Rates for Diesel Generators: Estimates Based on Licensee Event Reports at U.S. Commercial Nuclear Power Plants 1976-1978, NUREG/CR2099, EGG-EA-5359, Rev. 1.

Atwood, Corwin L., 1983, Data Analysis Using the Binomial Failure Rate Common Cause Model, NUREG/CR-3437; EGG-2271, p. 11.

Atwood, Corwin L., 1994a, Hits per Trial: Basic Analysis of Binomial Data, EGG-RAAM-11041.

Atwood, Corwin L., 1994b, Constrained Noninformative Priors in Risk Assessment, INEL-94/00029, submitted for publication.

Atwood, Corwin L., 1995, Modeling Patterns in Count Data Using Loglinear and Related Models, INEL95/0121.

Box, George E. P., and Gwilym M. Jenkins, 1970, Time Series Analysis: Forecasting and Control, San Francisco: Holden-Day, p. 17.

Engelhardt, M. E., 1994, Events in Time: Basic Analysis of Poisson Data, EGG-RAAM-11088.

Engelhardt, M. E., 1995a, Modeling Patterns in Continuous Data Using Linear and Related Models DRAFT, 1995, INEL-95/0120.

Engelhardt, M. E., 1995b, Statistical Analysis of Random Duration Times DRAFT, 1995, INEL-95/0206.

Grant, G. M., W. S. Roesener, D. G. Hall, C. L. Atwood, C. D. Gentillon, and T. R. Wolf, 1995, High-Pressure Coolant Injection (HPCI) System Performance, 1987-1993, INEL-94/0158.

Long, J. Scott, ed., 1988, Common Problems/Proper Solutions: Avoiding Errors in Quantitative Research, 2111 West Hillcrest Dr., Newbury Park, CA 91320: SAGE Publications. 
Martz, H. F. and R. A. Waller, 1990, "Reliability Analysis of Complex Series/Parallel Systems of Binomial Subsystems and Components," Technometrics, 32, pp. 407-416.

Meachum, Teresa R., and Corwin L. Atwood, 1983, Common Cause Fault Rates for Instrumentation and Control Assemblies, NUREG/CR-3289, EGG-2258.

Proschan, Frank, 1963, “Theoretical Explanation of Observed Decreasing Failure Rate," Technometrics, 5, pp. 375-383.

Roesener, W. S., G. M. Grant, and C. D. Gentillon, 1995, High Pressure Core Spray (HPCS) System Performance, 1987-1993, INEL-95/0133.

SAS Institute Inc., 1988, SAS/STAT User's Guide, Release 6.03 Edition, Cary, NC.

SAS Institute Inc., 1993, SAS/STAT Software: The GENMOD Procedure, Release 6.09, SAS Technical Report P-243, Cary, NC.

Tukey, John W., 1961, “Discussion, Emphasizing the Connection Between Analysis of Variance and Spectrum Analysis," Technometrics, 3, p. 202. 
APPENDIX A

SAS PROGRAMS

A-1 


$$
\text { A-2 }
$$




\section{APPENDIX A}

\section{SAS PROGRAMS}

This appendix gives SAS programs for finding the moments of various combinations of failures, in terms of the moments of the more fundamental failures, and programs relating the moments to the parameters of a beta distribution. All the programs are macros, and are intended to be invoked from within a SAS data step.

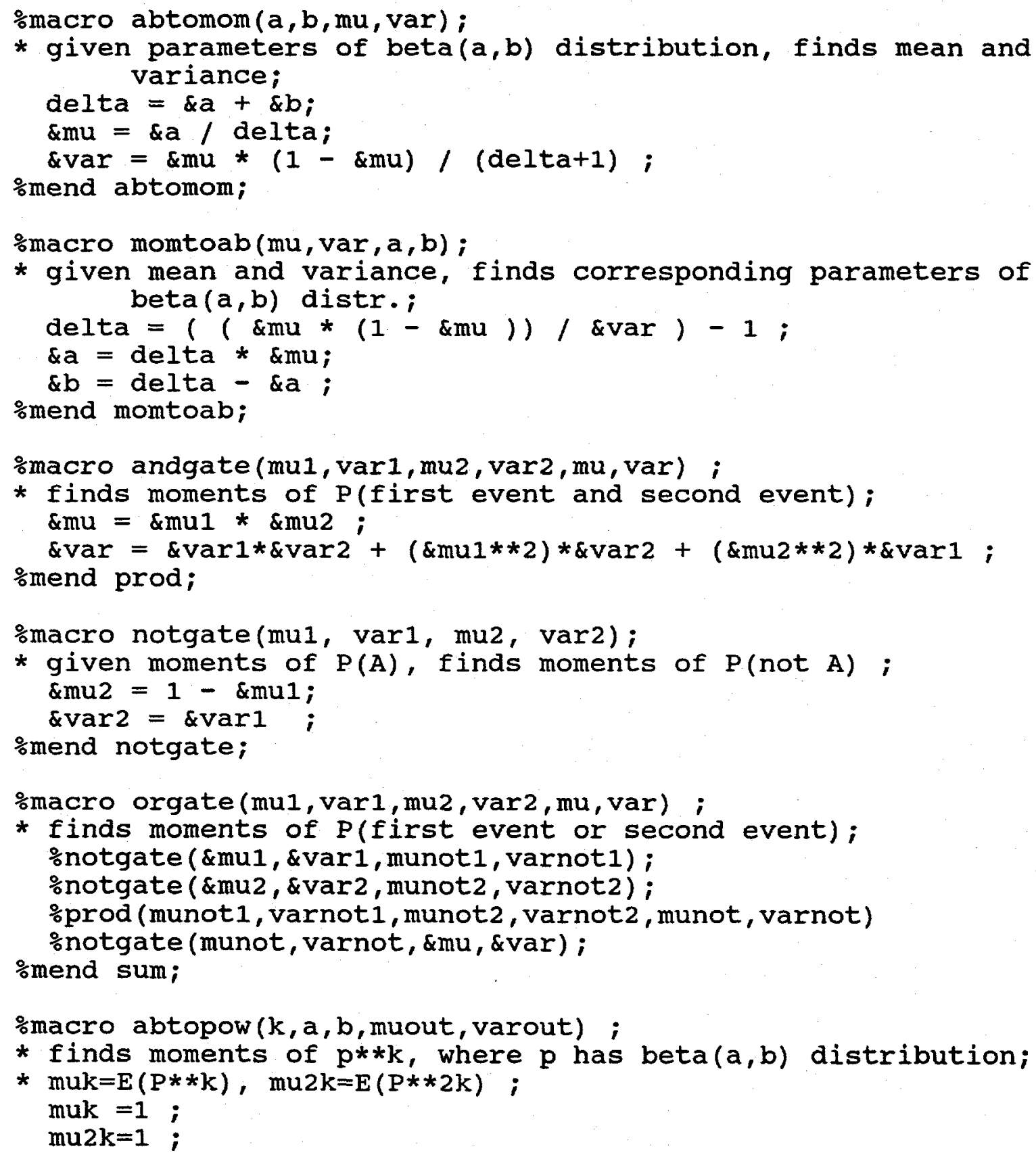




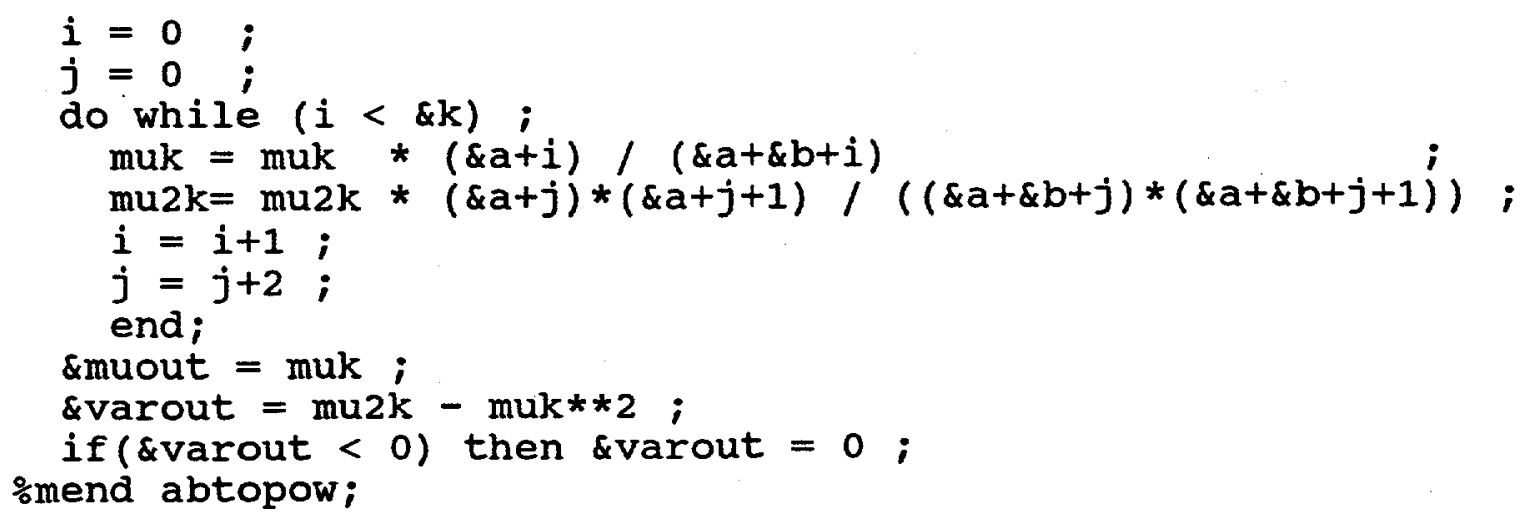




\section{APPENDIX B}

\section{LIKELIHOOD FORMULAS}


B-2 


\section{APPENDIX B}

\section{LIKELIHOOD FORMULAS}

The use of likelihoods suggested in Section 4.2.4 requires formulas for the likelihoods. A problem can arise when the formulas are used in computation, because the individual terms in the likelihoods can be very large. Although these large terms cancel each other, attempting to compute them individually can lead to numerical overflow. In particular, the factorial function, $n$ !, is enormous for moderate values of $n$. Therefore, it is most practical to work with logarithms, which take smaller values. As part of this, express the factorial in terms of $\ln \Gamma$, the logarithm of the gamma function, where the gamma function satisfies $\Gamma(n+1)=n$ ! Many software packages, including SAS, have $\ln \Gamma$ as a built-in function.

The logarithm of a likelihood is called a loglikelihood. The loglikelihoods are given here for some standard distributions. They can normally be calculated without numerical problems.

Let $X$ have a binomial $(n, p)$ distribution, and suppose that $X$ takes the value $x$. The corresponding loglikelihood, denoted $L_{\text {birom }}(p ; x)$, is

$$
\begin{aligned}
L_{b i n o m}(p ; x) & =\ln (n !)-\ln (x !)-\ln [(n-x) !]+x \ln (p)+(n-x) \ln (1-p) \\
& =\ln \Gamma(n+1)-\ln \Gamma(x+1)-\ln \Gamma(n-x+1)+x \ln (p)+(n-x) \ln (1-p)
\end{aligned}
$$

When several independent random variables $X_{i}$ have binomial $\left(n_{i}, p_{i}\right)$ distributions, the loglikelihood is

$$
L_{\text {binom }}\left(p_{1}, p_{2}, \ldots ; x_{1}, x_{2}, \ldots\right)=\sum_{i} L_{\text {binom }}\left(p_{i} ; x_{i}\right)
$$

In particular, if the $X_{i}$ s are assumed to have a common value of $p$, then every $p_{i}$ is replaced by $p$. Now suppose instead that each $p_{i}$ is drawn from a beta $(\alpha, \beta)$ distribution. Then each random variable $X_{i}$ has a beta-binomial $\left(n_{i}, \alpha, \beta\right)$ distribution, and the loglikelihood is

$$
\begin{aligned}
& L_{\text {beta-binom }}\left(\alpha, \beta ; x_{1}, x_{2}, \ldots\right) \\
& =\sum_{i}\left[\ln \Gamma\left(\alpha+x_{i}\right)-\ln \Gamma(\alpha)+\ln \Gamma\left(\beta+n_{i}-x_{i}\right)-\ln \Gamma(\beta)+\ln \Gamma(\alpha+\beta)-\ln \Gamma\left(\alpha+\beta+n_{i}\right)\right. \\
& \left.\quad+\ln \Gamma\left(n_{i}+1\right)-\ln \Gamma\left(x_{i}+1\right)-\ln \Gamma\left(n_{i}-x_{i}+1\right)\right] \\
& =\sum_{i}\left[\sum_{j=0}^{x_{i}-1} \ln (\alpha+j)+\sum_{j=0}^{n_{i}-x_{i}-1} \ln (\beta+j)-\sum_{j=0}^{n_{i}-1} \ln (\alpha+\beta+j)+\ln \Gamma\left(n_{i}+1\right)-\ln \Gamma\left(x_{i}+1\right)-\ln \Gamma\left(n_{i}-x_{i}+1\right)\right]
\end{aligned}
$$


All these formulas are based on standard results, as given, for example, by Atwood (1994).

Consider now events in time. Let $X$ be a count of events occurring with rate $\lambda$ in time $t$, so that $X$ is Poisson $(\lambda t)$. Then the loglikelihood, denoted $L_{\text {Poison }}(\lambda ; x)$ is

$L_{\text {Poieson }}(\lambda ; x)=x \ln (\lambda t)-\lambda t-\ln \Gamma(x+1)$

If, independent random variables $X_{i}$ are Poisson counts corresponding to rates $\lambda_{i}$ in times $t_{i}$, the likelihood is

$L_{\text {Poisson }}\left(\lambda_{1}, \lambda_{2}, \ldots ; x_{1}, x_{2}, \ldots\right)=\sum_{i} L_{\text {Poisson }}\left(\lambda_{i} ; x_{i}\right)$

In particular, if all the rates are assumed to be equal, each $\lambda_{i}$ is replaced by $\lambda$. If, instead, each $\lambda_{i}$ is drawn from a gamma $(\alpha, \beta)$ distribution, as described by Engelhardt (1994), then $X_{i}$ has a gamma-Poisson distribution, which can also be shown to be a negative binomial distribution. The loglikelihood is

$$
\begin{aligned}
& L_{\text {gamma-Poisson }}\left(\alpha, \beta ; x_{1}, x_{2}, \ldots\right) \\
& \quad=\sum_{i}\left[\ln \Gamma\left(\alpha+x_{i}\right)-\ln \Gamma(\alpha)-\ln \left(x_{i} !\right)+x_{i} \ln \left(t_{i} / \beta\right)-\left(\alpha+x_{i}\right)\left(1+t_{i} / \beta\right)\right] \\
& \quad=\sum_{i}\left[\sum_{j=0}^{x_{i}-1} \ln (\alpha+j)-\ln \Gamma\left(x_{i}+1\right)+x_{i} \ln \left(t_{i} / \beta\right)-\left(\alpha+x_{i}\right)\left(1+t_{i} / \beta\right)\right] .
\end{aligned}
$$

\section{REFERENCES}

Atwood, Corwin L., 1994, Hits per Trial: Basic Analysis of Binomial Data, EGG-RAAM-11041.

Engelhardt, M. E., 1994, Events in Time: Basic Analysis of Poisson Data, EGG-RAAM-11088. 